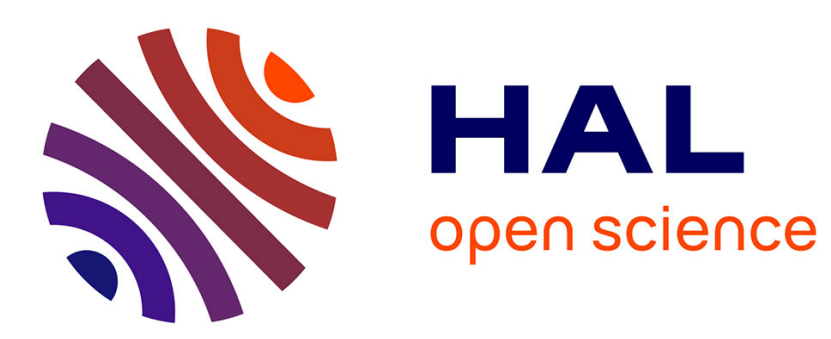

\title{
Wave patterns in film flows: Modelling and three-dimensional waves
}

B. Scheid, C. Ruyer-Quil, Paul Manneville

\section{To cite this version:}

B. Scheid, C. Ruyer-Quil, Paul Manneville. Wave patterns in film flows: Modelling and three-dimensional waves. Journal of Fluid Mechanics, 2006, 562 (september), pp.183-222. 10.1017/s0022112006000978 . hal-01023374

\section{HAL Id: hal-01023374 \\ https://hal-polytechnique.archives-ouvertes.fr/hal-01023374}

Submitted on 28 Jul 2014

HAL is a multi-disciplinary open access archive for the deposit and dissemination of scientific research documents, whether they are published or not. The documents may come from teaching and research institutions in France or abroad, or from public or private research centers.
L'archive ouverte pluridisciplinaire HAL, est destinée au dépôt et à la diffusion de documents scientifiques de niveau recherche, publiés ou non, émanant des établissements d'enseignement et de recherche français ou étrangers, des laboratoires publics ou privés. 


\title{
Wave patterns in film flows: modelling and three-dimensional waves
}

\author{
By BENOIT SCHEID ${ }^{1}$, CHRISTIAN RUYER-QUIL ${ }^{2}$ \\ AND PAUL MANNEVILLE ${ }^{3}$ \\ ${ }^{1}$ Service de Chimie-Physique E.P., Université Libre de Bruxelles, C.P. 165/62, 1050 Brussels, Belgium \\ ${ }^{2}$ Laboratoire FAST - UMR CNRS 7608, Campus universitaire, 91405 Orsay, France \\ ${ }^{3}$ LadHyX - UMR CNRS 7646, École polytechnique, 91128 Palaiseau, France
}

(Received 17 June 2005 and in revised form 25 January 2006)

In a previous work, two-dimensional film flows were modelled using a weightedresidual approach that led to a four-equation model consistent at order $\epsilon^{2}$. A twoequation model resulted from a subsequent simplification but at the cost of lowering the degree of the approximation to order $\epsilon$ only. A Padé approximant technique is applied here to derive a refined two-equation model consistent at order $\epsilon^{2}$. This model, formulated in terms of coupled evolution equations for the film thickness $h$ and the flow rate $q$, accounts for inertia effects due to the deviations of the velocity profile from the parabolic shape, and closely follows the asymptotic long-wave expansion in the appropriate limit. Comparisons of two-dimensional wave properties with experiments and direct numerical simulations show good agreement for the range of parameters in which a two-dimensional wavy motion is reported in experiments.

The stability of two-dimensional travelling waves to three-dimensional perturbations is investigated based on the extension of the models to include spanwise dependence. The secondary instability is found to be not very selective, which explains the widespread presence of the synchronous instability observed in the experiments by Liu et al. (1995) whereas Floquet analysis predicts a subharmonic scenario in most cases. Three-dimensional wave patterns are computed next assuming periodic boundary conditions. Transition from two- to three-dimensional flows is shown to be strongly dependent on initial conditions. The herringbone patterns, the synchronously deformed fronts and the three-dimensional solitary waves observed in experiments are recovered using our regularized model, which is found to be an excellent compromise between the complete model, which has seven equations, and the simplified model, which does not include the second-order inertia corrections. Those corrections are found to play a role in the selection of the type of secondary instability as well as of the spanwise wavelength of the emerging pattern.

\section{Introduction}

Thin films flowing down inclines have a rich dynamics, extensively studied for a long time since Kapitza's experimental and theoretical pioneering work at the end of the 1940s (Kapitza 1948; Kapitza \& Kapitza 1949). Most of the experimental studies devoted to this problem are referred to in the book by Alekseenko, Nakoryakov \& Pokusaev (1994). More recent experimental results are presented for example in Nosoko et al. (1996), Vlachogiannis \& Bontozoglou (2001), Park \& Nosoko (2003), Nosoko \& Miyara (2004), and Argyriadi, Serifi \& Bontozoglou (2004). At Haverford, 
Gollub and coworkers have performed an extensive study of water-glycerin mixtures flowing down weakly inclined planes, see Liu \& Gollub (1993), Liu, Paul \& Gollub (1993), Liu \& Gollub (1994), and Liu, Schneider \& Gollub (1995). Controlling the entrance flow rate, they applied a periodic forcing at the inlet and observed the response of the film at a given frequency. Their experiments give the clearest picture of the phenomenology of waves on film flows. At frequencies close to but below the cut-off frequency $f_{c}$, the primary instability gives rise to saturated two-dimensional waves. $\uparrow$ These waves are slow and present wide bumpy crests and deep thin troughs. They belong to the $\gamma_{1}$ family in the terminology introduced by Chang, Demekhin \& Kopelevitch (1993). At low frequencies, large-amplitude solitary waves in the form of fast humps preceded by small capillary ripples emerge from the inception region. Such waves belong to the $\gamma_{2}$ family. By identifying the different secondary instabilities of the saturated two-dimensional waves leading to disorder, the observations of the Haverford group complete the review by Chang (1994).

The purpose of this paper is to propose an accurate model able to account for the experiments by Liu et al. (1995) and ultimately obtain a unified theoretical understanding of the experimental data available in the literature. The separation of scales implied by the long-wave character of the instability allows one to define a small parameter $\epsilon$, called the film parameter, basically measuring the slope of the interface in order of magnitude, and to apply Prandtl's simplification of the crossstream momentum equation, usual in boundary layer theory, which helps one to eliminate the in-depth pressure distribution dominated here by surface tension and gravity. This leads to so-called boundary-layer equations, see Chang et al. (1993) for a detailed presentation. These equations can be viewed as the first step of the long-wave expansion performed by Benney (1966). Modulations of the film thickness around the flat-film solution being slow in space and time, the product of the film parameter $\epsilon$ and the Reynolds number $R$ is small as in classical lubrication theory. Inertia is thus small and consequently the velocity field stays enslaved to the film thickness. This leads to a single evolution equation for the film thickness $h$ governing the dynamics of the flow at the onset of the instability. Several one-equation models have therefore been proposed to investigate the three-dimensional dynamics of film flows (Roskes 1969; Atherton \& Homsy 1976; Roy, Roberts \& Simpson 2002; Saprykin, Demekhin \& Kalliadasis 2005). However, for the range of Reynolds numbers where three-dimensional wavy regimes have been reported by Liu et al. (1995) and Park \& Nosoko (2003), one-equation models have been shown to fail, either leading to an underestimation of the wave speeds and heights, or exhibiting unphysical behaviours (Pumir, Manneville \& Pomeau 1983; Ooshida 1999; Scheid et al. 2005b).

An alternative to the gradient expansion approach is to make use of the KármánPolhausen averaging technique as in boundary-layer theory (Schlichting 1955). This technique, which was first proposed by Kapitza (1948) and later re-investigated by Shkadov (1967), leads to a two-field model involving the film thickness $h$ and the local flow rate $q$, for which the velocity field is not taken to be entirely enslaved to the film thickness. In both cases, a reduction of the dimensionality of the basic equations is achieved through the elimination of the cross-stream coordinate. The transition of film flows to three-dimensional dynamics was first theoretically investigated in this

$\dagger$ Two- vs. three-dimensional refers to the fluid velocity dependence. Two-dimensional flow means spanwise independent (coordinates $x$ and $y$ ) while the surface elevation is one-dimensionally modulated (along $x$ ). On the other hand, full three-dimensional flow $(x, y, z)$ involves twodimensional thickness modulations $(x, z)$. 
context by Trifonov (1989). Starting from two-dimensional solutions to the KapitzaShkadov model computed at rest in a moving frame, he analysed their stability to transverse modulations and showed that the subharmonic instability was always the most dangerous one. The stationary three-dimensional waves bifurcating from the two-dimensional waves of the $\gamma_{1}$ family were shown to have transverse modulations with troughs that deepen faster than the peaks grow, which eventually produced trains of isolated depressions, as experimentally observed by Liu et al. (1995). Chang et al. (1994) attempted to complete Trifonov's study by using the boundary-layer equations. Their stability analysis of the $\gamma_{1}$ family predicted only the subharmonic instability, hence a scenario different from the one reported by Liu \& Gollub, i.e. not accounting for the presence of the synchronous mode. Trifonov and Chang et al. both only considered vertical walls whereas the experiments at Haverford were performed for an inclined wall where hydrostatic pressure plays a significant role. To our knowledge, there is as yet no thorough theoretical understanding of the full experimental results and especially of the three-dimensional synchronous instability of the slow saturated $\gamma_{1}$ waves.

The basic set of equations and boundary conditions governing the problem is given in $\S 2.1$, followed by a presentation of the boundary layer approximation in $\S 2.2$. From $\S 3$ to $\S 5$, two-dimensional flows are considered, whereas three-dimensional flows are investigated in $\S 6$ to $\S 8$. Section 3 is devoted to a short presentation of the regularization method introduced by Ooshida (1999) to film flows. In $\S 4$, we start discussing our previous extension (Ruyer-Quil \& Manneville 2000) of Shkadov's approach (Shkadov 1967) (\$4.1). An adiabatic elimination of velocity corrections (§4.2 and $\S 4.3)$ is next followed by an algebraic Padé-like approach $(\S 4.4)$ aiming at a model accurate at order $\epsilon^{2}$ that does not suffer from the previous limitations. The quantitative validation of the models in the two-dimensional wavy regime is considered in $\S 5$. In $\S 6$, we extend our models to three-dimensional flows. In $\S 7$, we develop a standard Floquet stability analysis of the $\gamma_{1}$ waves corresponding to the experiments by Liu et al. (1995). Section 8 is dedicated to the numerical simulations of the models and a comparison with various experimental data existing in the literature. We first concentrate on the selection of the different three-dimensional wave patterns resulting from the streamwise-periodic forcing of $\gamma_{1}$ waves reported by Liu et al. (1995) (\$ 8.1). The sensitivity to initial conditions is discussed and the results of the different models are compared. We next use the regularized model to study the three-dimensional instability of $\gamma_{2}$ waves corresponding to the experimental work by Park \& Nosoko (2003) in $§ 8.2$. Finally, the development of natural (i.e. noise-driven) three-dimensional waves is investigated, from two-dimensional wave trains to threedimensional solitary waves, and compared to the experimental data by Alekseenko et al. (1994) in $\S 8.3$. Concluding remarks and perspectives are presented in $\S 9$.

\section{Governing equations}

\subsection{Primitive equations}

The flow of a Newtonian liquid down a plane making an angle $\beta$ with the horizontal is considered. Coordinate $x$ defines the streamwise direction, $y$ denotes the direction normal to the plane, and $z$ is along the spanwise direction (unit vectors $\boldsymbol{i}, \boldsymbol{j}, \boldsymbol{k}$ respectively); $\boldsymbol{u} \equiv u \boldsymbol{i}+v \boldsymbol{j}+w \boldsymbol{k}$ is the velocity field and $p$ is the pressure. Surface tension $\sigma$, viscosity $\mu$, and density $\rho$, are assumed to remain constant. The dimensionless form of the governing equations is obtained with length and time scales based on the kinematic viscosity $v=\mu / \rho$ and the streamwise gravitational acceleration 
$g \sin \beta$ so that they depend only on the physical properties of the fluid and the inclination angle. They are

$$
l_{v}=v^{2 / 3}(g \sin \beta)^{-1 / 3} \quad \text { and } \quad t_{v}=v^{1 / 3}(g \sin \beta)^{-2 / 3} .
$$

This scaling is appropriate provided that $\sin \beta \sim O(1)$, i.e. excluding near-horizontal configurations, for which instabilities that set in are typical of wall flows, involving Tollmien-Schlichting waves of shear-viscous origin, see e.g. Floryan, Davis \& Kelly (1987). The flow conditions can further be characterized by the dimensionless thickness of the flat film solution (Nusselt flow), $h_{\mathrm{N}}$, the inclination $B=\cot \beta$ and the Kapitza number $\Gamma=\sigma /\left[\rho v^{4 / 3}(g \sin \beta)^{1 / 3}\right]$ which compares the surface stress $\sigma / l_{v}$ to the viscous stress $\mu / t_{v}$. Using these scales, the Navier-Stokes equation is

$$
\partial_{t} \boldsymbol{u}+\boldsymbol{u} \cdot \nabla \boldsymbol{u}=\boldsymbol{i}-B \boldsymbol{j}-\nabla p+\nabla^{2} \boldsymbol{u} .
$$

Above and in the following, $\partial_{\alpha}$ denotes partial differentiation with respect to variable $\alpha$. The continuity equation for an incompressible flow is

$$
\nabla \cdot \boldsymbol{u}=0 .
$$

The evolution equations need to be supplemented with boundary conditions at the bottom plane, $y=0$, and at the free surface, $y=h$. A quantity $\beta$ evaluated at $y=\tilde{y}$ will be denoted by $\left.\beta\right|_{\tilde{y}}$. The flow is thus subjected to the usual no-slip condition:

$$
\left.\boldsymbol{u}\right|_{0}=0 \text {. }
$$

The interface is governed by the kinematic condition expressing that the free surface is a material surface, that is

$$
\left(\partial_{t}+\boldsymbol{u} \cdot \nabla\right)(h(x, z, t)-y)=0
$$

or

$$
\left.v\right|_{h}=\left(\partial_{t}+\left.u\right|_{h} \partial_{x}+\left.w\right|_{h} \partial_{z}\right) h
$$

Finally, the stress balance at the interface is

$$
-p \boldsymbol{n}+\left(\nabla \boldsymbol{u}+\nabla \boldsymbol{u}^{T}\right) \cdot \boldsymbol{n}=-\Gamma(\nabla \cdot \boldsymbol{n}) \boldsymbol{n},
$$

where $\boldsymbol{n}$ is the unit vector normal to the free surface oriented outwards.

Alternatively, Reynolds and Weber numbers based on the entrance flow rate are often preferred though they do not clearly separate flow conditions from the fluid's physical constants. The relations between these dimensionless parameters are easily obtained by noticing that, at the entrance, the interface is flat so that the Reynolds number is related to the dimensionless Nusselt thickness $h_{\mathrm{N}}$ through an integration of the parabolic velocity profile $u \equiv y\left(h_{\mathrm{N}}-\frac{1}{2} y^{2}\right)$ over the depth. This gives

$$
R \equiv q_{\mathrm{N}}=\frac{1}{3} h_{\mathrm{N}}^{3},
$$

where $q_{\mathrm{N}}$ is the dimensionless Nusselt flow rate. Similarly, the Weber number is related to the Kapitza number through

$$
W=\Gamma h_{\mathrm{N}}{ }^{-2} .
$$

\subsection{Lubrication approximation and Shkadov's scaling}

Considering slow space and time variation, the formal parameter $\epsilon$ is introduced along with each derivation in space or time $\partial_{x, z, t} \propto \epsilon$. The assumed slow space variation implies that the velocity component normal to the plane $v$ is much smaller than 
the streamwise and spanwise components $u$ and $w$ as derived from the continuity equation (2.2). Consequently, the inertia terms in the $y$-component of the momentum equation are of higher order and can be dropped out. The remaining equation is then linear and can be integrated to give the pressure distribution up to order $\epsilon$. After substitution of the latter and some algebra detailed in Ruyer-Quil \& Manneville (1998), approximated streamwise and spanwise momentum equations are obtained.

At this stage it is convenient to proceed to the rescaling of space variables introduced by Shkadov (1977). At a given inlet flow rate, the natural scale for $y$ is the Nusselt flat film thickness $h_{\mathrm{N}}$, which yields the changes $(y, h)=\left(h_{\mathrm{N}} \tilde{y}, h_{\mathrm{N}} \tilde{h}\right)$. Then balancing gravity forces and surface tension introduces the scale ratio $\kappa=\left(\Gamma / h_{\mathrm{N}}^{2}\right)^{1 / 3} \equiv W^{1 / 3}$. Shkadov proceeded therefore to a compression of the streamwise and spanwise coordinates and took the scale for $x$ and $z$ as $\kappa$ times the scale for $y$, hence the changes $x=\kappa h_{\mathrm{N}} \tilde{x}$ and $z=\kappa h_{\mathrm{N}} \tilde{z}$. Scaling time as $t=\left(\kappa / h_{\mathrm{N}}\right) \tilde{t}$ and velocity components as $u=h_{\mathrm{N}}^{2} \tilde{u}, w=h_{\mathrm{N}}^{2} \tilde{w}$ and $v=\left(h_{\mathrm{N}}^{2} / \kappa\right) \tilde{v}$, and dropping tildes, the rescaled streamwise momentum equation is

$$
\begin{aligned}
\delta\left[\partial_{t} u+\partial_{x}\left(u^{2}\right)+\partial_{y}(u v)+\partial_{z}(u w)\right]= & 1+\partial_{y y} u-\zeta \partial_{x} h+\partial_{x x x} h+\partial_{x z z} h \\
& +\eta\left[2 \partial_{x x} u+\partial_{z z} u+\partial_{x z} w-\partial_{x}\left(\left.\partial_{y} v\right|_{h}\right)\right],
\end{aligned}
$$

where

$$
\delta=h_{\mathrm{N}}^{3} / \kappa=3 R W^{-1 / 3}
$$

is a reduced Reynolds number. The two other reduced parameters

$$
\zeta=B / \kappa=\cot \beta W^{-1 / 3} \quad \text { and } \quad \eta=\kappa^{-2}=W^{-2 / 3}
$$

respectively measure the effect of the gravity component normal to the plane and the viscous second-order effects. The reduced Reynolds number introduced by Shkadov was $\delta / 45$; the present choice is preferred since it leaves all numerical coefficients in the equations unchanged.

Except for the presence in (2.8) of the gravity term scaled to unity, the streamwise and spanwise momentum equations are symmetric under the exchange $\{u \leftrightarrow w$, $x \leftrightarrow z\}$. The rescaling of our set of equations leave the no-slip condition (2.3) and the kinematic condition (2.4) unchanged, whereas the stress balance at the free surface and in the $x$-direction is now at $O\left(\epsilon^{2}\right)$

$$
\partial_{y} u=\eta\left[\partial_{z} h\left(\partial_{z} u+\partial_{x} w\right)+2 \partial_{x} h\left(2 \partial_{x} u+\partial_{z} w\right)-\partial_{x} v\right] \quad \text { at } y=h .
$$

The set of boundary conditions is then closed by the stress balance in the $z$-direction obtained from (2.11) through the exchange $\{u \leftrightarrow w, x \leftrightarrow z\}$. The set of equations obtained are usually referred as the second-order boundary-layer equations since the assumptions leading to them are essentially the same as those in the derivation of the Prandtl equation of boundary-layer theory, see Schlichting (1955). Within our basic assumptions, they are consistent at order $\epsilon^{2}$.

The set of reduced parameters $\delta, \zeta$ and $\eta$ is formally equivalent to the set $R, B$ and $W\left(\right.$ or $h_{\mathrm{N}}, B, \Gamma$ ). An advantage of Shkadov's scaling is that it collects all second-order viscous terms into the sole parameter $\eta$. Since these terms are the only physical ones of order $\epsilon^{2}$ in equations (2.8), (2.11), the truncation of the boundary-layer equations at first order leaves $\delta$ as the only parameter, provided that the wall is vertical $(\zeta=0)$, as was the case in many studies. 


\section{One-equation reduction and Padé-like regularization}

Comparisons between existing models and the subsequent discussion about improvements needed can be made simpler if the spanwise dependence of the fields is disregarded. Accordingly, from this section up to $\S 6$, we focus on two-dimensional flows $\left(\partial_{z} \equiv 0, w \equiv 0\right)$.

A gradient expansion of the basic equations or the boundary-layer equations leads to identical results up to order $\epsilon^{2}$. Such an expansion of the basic equations was first done by Benney (1966) and next completed by Lin (1974) and Nakaya (1975). Benney showed that the velocity field $\boldsymbol{u}$ can be written as a series of polynomials in $y$, i.e. $\boldsymbol{u}=\sum_{n} \boldsymbol{A}_{n}(h) P_{n}(y)$, where the coefficients $\boldsymbol{A}_{n}$ are functions of the thickness $h$ and its space-time derivatives, which means that, in this limit, the velocity field is completely enslaved to the dynamics of $h$. Integration of the continuity equation across the layer leads to the exact mass balance equation:

$$
\partial_{t} h+\partial_{x} q=0,
$$

where $q=\int_{0}^{h} u \mathrm{~d} y$ is the local flow rate. The gradient expansion of the momentum balance equation next gives an approximate expression for the flow rate as function of $h$ and its derivatives. This expression can be further simplified by using the zerothorder relation $q^{(0)}=\frac{1}{3} h^{3}$ to exchange the time derivative of $h$ with its space derivative through

$$
\partial_{t} h=-h^{2} \partial_{x} h
$$

which is the equation governing kinematic waves at the interface (Whitham 1974). Gjevik $(1970,1971)$ thus studied the following equation:

$$
\partial_{t} h+\frac{1}{3} \partial_{x}\left\{h^{3}+\frac{2}{35} \delta \partial_{x}\left(h^{7}\right)-\frac{1}{4} \zeta \partial_{x}\left(h^{4}\right)+h^{3} \partial_{x x x} h\right\}=0,
$$

generally called the Benney equation.

The relevance of this equation beyond a narrow neighbourhood of the threshold is first limited by the fact that linear stability properties of the flat film solution rapidly depart from those derived from the exact Orr-Sommerfeld (OS) equation, i.e. the range of unstable wavenumbers predicted by (3.3) is much wider than that emerging from the solution of the OS equation. This first limitation seems related to the neglect of the second-order streamwise dissipative terms as shown by Panga \& Balakotaiah (2003). Taking only them into account, Panga \& Balakotaiah obtained an equation which, within current scalings, is

$$
\begin{aligned}
\partial_{t} h+\frac{1}{3} \partial_{x}\left\{h^{3}-\frac{1}{8} \delta \partial_{t}\left(h^{5}\right)-\frac{9}{280} \delta \partial_{x}\left(h^{7}\right)-\frac{1}{4} \zeta \partial_{x}\left(h^{4}\right)+h^{3} \partial_{x x x} h\right. \\
\left.+\eta\left[3 h^{4} \partial_{x x} h+7 h^{3}\left(\partial_{x} h\right)^{2}\right]\right\}=0 .
\end{aligned}
$$

Panga \& Balakotaiah avoided the exchange of the time and space derivatives through (3.2) and showed that the exact OS results are then recovered with better accuracy. Unfortunately, this correction does not cure the second well-known limitation of the Benney equation (3.3), that is, the existence of finite-time blow-up of its solutions beyond some limiting value of the Reynolds number not far beyond threshold (Pumir et al. 1983; Scheid et al. 2005b) since (3.4) also suffers from finite-time blow-up of solutions somewhat beyond threshold (Ruyer-Quil \& Manneville 2004). Pumir et al. (1983) showed in particular that the finite-time blow-up of time-dependent solutions closely corresponds to the loss of one-hump solitary waves, i.e. homoclinic orbits in the terminology of dynamical systems theory. Our experience with similar but more complicated equations (Ruyer-Quil 1999) suggests this that loss of what is called the 
'principal homoclinic orbit' by Glendinning \& Sparrow (1984) is accompanied by a blow-up of time-dependent solutions.

In order to remedy this deficiency, Ooshida (1999) developed a resummation method inspired by the Padé approximant technique. The latter relies on the idea that the divergence of a power series $Q=\sum_{k} Q_{k} x^{k}$ is due to the hidden presence of poles. This leads one to express $Q$ in an approximate way as a ratio $F / G$ of polynomials $F$ and $G$ where the zeros of $G$ are assumed to capture the causes of the divergence. Adjusting the coefficients introduced in $F=F_{0}+F_{1} x+F_{2} x^{2} \ldots$ and $G=1+G_{1} x+G_{2} x^{2}+\ldots$ so that the terms in the series $Q$ are reproduced exactly up to some given degree is the essence of the approximation, the ratio $F / G$ being used in place of $Q$. In this algebraic implementation, the degrees of the polynomials $F$ and $G$ are open to free choice, the number of coefficients to be determined remaining compatible with the number of coefficients available in the series $Q$.

Ooshida translated this idea to the present case by introducing a regularization operator $\mathscr{G}=\mathscr{I}+\mathscr{G}^{(1)}+\mathscr{G}^{(2)}$, where $\mathscr{I}$ is the identity, $\mathscr{G}^{(1)}=G^{(1)}(h) \partial_{x}$, and $\mathscr{G}^{(2)}=$ $G^{(2)}(h) \partial_{x x}$, so that the expansion of $q$ as a function of $h$ and its derivatives from the long-wave expansion, formally written as $q \equiv \mathscr{Q}(h)$, is rewritten as $\mathscr{G}^{-1} \mathscr{F}$. Ooshida chose to adjust 'coefficients' $\mathscr{G}^{(1)}$ and $\mathscr{G}^{(2)}$ in $\mathscr{G}$ so that $\mathscr{G} \mathscr{Q}=\mathscr{F}$ could be reduced to $q^{(0)}+\mathscr{F}^{(1)}$, i.e. $\mathscr{F}^{(2)} \equiv 0$, which yielded

$$
\mathscr{G}=1-\frac{10}{21} \delta h^{4} \partial_{x}-\eta h^{2} \partial_{x x}
$$

Computation of the regularized identity $\partial_{x}(\mathscr{G} \mathscr{Q}) \equiv \partial_{x} \mathscr{F}$ with the replacement of $\partial_{x} \mathscr{Q}$ by $-\partial_{t} h$ using (3.1) led him to the equation

$$
\partial_{t} h+\frac{1}{3} \partial_{x}\left\{h^{3}-3 \eta h^{2} \partial_{x t} h-\frac{2}{7} \delta \partial_{t}\left(h^{5}\right)-\frac{36}{245} \delta \partial_{x}\left(h^{7}\right)-\frac{1}{4} \zeta \partial_{x}\left(h^{4}\right)+h^{3} \partial_{x x x} h\right\}=0 .
$$

Ooshida's formulation remedies the possible blow-up of time-dependent solutions observed with (3.3) but (3.5) grossly underestimates the amplitudes and speeds of the solitary waves. Panga, Mudunuri \& Balakotaiah (2005) attempted to apply Ooshida's idea to regularize equation (3.4) which led them to an expression for $q$ as function of $h$ and $\partial_{t} q$, which can be recast as an evolution equation for $q$ :

$$
\delta \partial_{t} q=\frac{8}{5} h-\frac{24}{5} \frac{q}{h^{2}}-\frac{9}{25} \delta h^{4} \partial_{x} h-\frac{8}{5} \zeta h \partial_{x} h+\frac{8}{5} h \partial_{x x x} h+\eta\left[\frac{56}{5} h\left(\partial_{x} h\right)^{2}+\frac{24}{5} h^{2} \partial_{x x} h\right] .
$$

Equation (3.6) must be completed by the mass conservation equation (3.1) and is referred to hereafter as the PMB model. As a consequence, the flow rate $q$ is no longer slaved to the evolution of the thickness $h$ which indicates that $q$ must be recognized as an independent degree of freedom (Balakotaiah \& Mudunuri 2004).

Once it is recognized that some freedom should be given back to the velocity field, this idea should be implemented from the beginning, which calls for a different approach if we require accurate modelling in the largest possible range of Reynolds numbers and not only in the neighbourhood of the instability threshold, i.e. also in what Ooshida called the 'drag-inertia' regime that takes place when inertia plays a more significant role at large $\delta$, as opposed to the 'drag-gravity' regime taking place at small $\delta$ and corresponding to a balance between viscous drag on the wall and gravitational acceleration, for which the classical long-wave expansion is expected to be valid. 


\section{Weighted residual modelling}

\subsection{General formulation}

The difficulty with modelling in terms of a single equation is that keeping a single dependent variable, namely $h$, is not sufficient to account for the dynamics of the film, though the perturbations may well remain long wave. At every step of the asymptotic expansion, the velocity profile is assumed to have no dynamics of its own but to be strictly enslaved to $h$ by equations where the time dependence only comes through that of $h$. This is justified only as long as the evolution rate of velocity modes, of order unity due to the viscous damping over the thickness, can be considered as large when compared to the evolution rate of $h$, of order $\epsilon$. Beyond threshold ( $\epsilon$ finite) this assumption fails, which can be interpreted as a sign of a revolt of enslaved degrees of freedom. The dynamics of the flow can then no longer be described through the evolution of a single field for the film thickness and other variables must be considered, e.g. the local flow rate $q$, the stress at the wall, etc.

This discrepancy motivated two of us to re-investigate Shkadov's approach (Shkadov 1967) and pursue his original suggestion of expanding the velocity field on a polynomial basis (Ruyer-Quil \& Manneville 2000). The first term of this expansion was taken to be $g_{0}(y)=y-\frac{1}{2} y^{2}$, the flat-film parabolic velocity profile. We showed that first-order corrections to the parabolic velocity distribution could be described entirely with the help of only two more polynomials of degree four and six, $g_{1}$ and $g_{2}$, the definition of which are given in Appendix A. We next proceeded to a Galerkin projection retaining terms up to order $\epsilon^{2}$. Writing the streamwise momentum balance formally as $B L(u)=0$, the residuals are $\mathscr{R}_{i}(u)=\left\langle B L(u), g_{i}(y)\right\rangle$, where $\langle f, g\rangle=\int_{0}^{h} f g \mathrm{~d} y$ refers to the scalar product derived from the plain $\mathscr{L}^{2}$ norm. Setting the three residuals $\mathscr{R}_{i}(u)$ to zero formed a system of three evolution equations for the three unknowns $q, r$ and $s$, whose extension to the three-dimensional case is given in Appendix $\mathrm{C}$ as $(\mathrm{C} 1 a-c)$. System (C1) is completed with the mass balance (3.1), and referred hereafter as the complete second-order model.

The theoretical analysis and the numerical integration of models such as the complete model are indeed simpler than the corresponding study of the full NavierStokes problem, or even of the boundary-layer formulation. Handling the four fields of (3.1), (C 1) still remains a difficult task, and a reliable two-field formulation consistent at order $\epsilon^{2}$ would be welcome. At this stage setting $r$ and $s$ to zero in $\mathscr{R}_{0}$ lowers the order of the approximation. This procedure leads to a simplified averaged momentum equation

$$
\begin{aligned}
\delta \partial_{t} q= & \frac{5}{6} h-\frac{5}{2} \frac{q}{h^{2}}+\delta\left[\frac{9}{7} \frac{q^{2}}{h^{2}} \partial_{x} h-\frac{17}{7} \frac{q}{h} \partial_{x} q\right]-\frac{5}{6} \zeta h \partial_{x} h+\frac{5}{6} h \partial_{x x x} h \\
& +\eta\left[4 \frac{q}{h^{2}}\left(\partial_{x} h\right)^{2}-\frac{9}{2 h} \partial_{x} q \partial_{x} h-6 \frac{q}{h} \partial_{x x} h+\frac{9}{2} \partial_{x x} q\right] .
\end{aligned}
$$

The set of equations to be solved is next closed by the mass conservation equation (3.1). Our simplified model was shown to predict the correct linear stability threshold. However, contrary to the gradient expansion of the complete model, the gradient expansion of (4.1) failed to reproduce the exact expression of the flow rate $q$ as function of $h$ at order $\epsilon^{2}$. As a matter of fact, results differ only through the coefficient of the first inertia term, which is $\frac{212}{525}$ instead of the exact value $\frac{127}{315}$ (RuyerQuil \& Manneville 2000). One should not be fooled by the apparent smallness of the differences between these coefficients. As shown in the next subsection, if small 
numerical coefficients are associated with the second-order inertia terms, they contain nonlinearities of high order, the effects of which become noticeable for $\delta$ of order unity or higher.

We develop below a consistent elimination strategy for $r$ and $s$ aimed at a twoequation model taking an exact account of the gradient expansion up to order $\epsilon^{2}$.

\subsection{Reduction of the full second-order model}

A simple argument can be given here to justify the pertinence of the elimination of the corrections to the parabolic velocity distribution, $r$ and $s$. Since viscosity acts so as to ensure the in-depth coherence of the flow, fluctuations of the flow field varying rapidly in the wall-normal direction are efficiently damped by viscosity, so that $r$ and $s$ corresponding to high-degree polynomials should relax rapidly towards the values forced by the evolution of $h$ and $q$. This can be observed simply by linearizing system $(\mathrm{C} 1)$ around the Nusselt flow in the zero-wavenumber limit, that is, assuming no spatial variations. The mass balance (3.1) thus implies a constant thickness. Writing $q=1 / 3+\varepsilon \tilde{q}, r=\varepsilon \tilde{r}$ and $s=\varepsilon \tilde{s}$ where $\varepsilon \ll 1$, we obtain

$$
\delta \frac{\mathrm{d} \tilde{\boldsymbol{V}}}{\mathrm{d} t}=\boldsymbol{M} \tilde{\boldsymbol{V}},
$$

where $\tilde{\boldsymbol{V}}=(\tilde{q}, \tilde{r}, \tilde{s})^{t}$ and $\boldsymbol{M}$ is a $3 \times 3$ matrix whose eigenvalues $\lambda_{i}$ are respectively $-2.47,-22.3$, and -87.7 . Because of the large gap between $\lambda_{1}$ and $\left(\lambda_{2}, \lambda_{3}\right)$, it is obvious that, at low Reynolds number and provided that the long-wave assumption is valid, the dynamics of the flow is governed by the neutral mode associated with the free-surface elevation and the eigenmode corresponding to $\lambda_{1}$, with eigenvector $(\tilde{q}, \tilde{r}, \tilde{s})^{t}=\left(1.00,-1.3310^{-2}, 1.3810^{-4}\right)^{t}$. Consequently and given that the associated eigenvector is nearly aligned with the first vector of the natural basis, $r$ and $s$ are truly slaved to the dynamics of the thickness $h$ and the flow rate $q$, at least close to the threshold.

Having justified the elimination of $r$ and $s$, let us go back to its practical implementation. Fields $r$ and $s$ are corrections to the flat-film parabolic profile corresponding to $g_{0}$. So, they are at least first-order terms produced by the deformation of the free surface. In the first residual $\mathscr{R}_{0}$ associated with the weight $g_{0}, r$ and $s$ appear through inertia terms involving their space and time derivatives or through products with derivatives of $h$ and $q$, which are terms of order $\epsilon^{2}$. Indeed, the corrections to the velocity field cannot appear in $\mathscr{R}_{0}$ at lowest order since the evaluation of the viscous term $\int_{0}^{h} g_{0}(y / h) \partial_{y y} u \mathrm{~d} y$ yields $\left.\frac{1}{2} \partial_{y} u\right|_{y=h}-q / h^{2}$, owing to the definition of $q=\int_{0}^{h} u \mathrm{~d} y$, and that $\left.\frac{1}{2} \partial_{y} u\right|_{y=h}$ is already of order $\epsilon^{2}$, as seen from (2.11) that expresses the stress balance at the free surface.

At this stage, it remains to determine the expression for $r$ and $s$ as functions of $h, q$ and their derivatives truncated at order $\epsilon$. Such relations can easily be obtained by dropping all second-order terms from the two last residuals $\mathscr{R}_{1}$ and $\mathscr{R}_{2}$ and then solving for $r$ and $s$.

$$
\begin{aligned}
& r=\delta\left[\frac{1}{210} h^{2} \partial_{t} q-\frac{19}{1925} q^{2} \partial_{x} h+\frac{74}{5775} h q \partial_{x} q\right]+O\left(\epsilon^{2}\right), \\
& s=\delta\left[\frac{2}{5775} q^{2} \partial_{x} h-\frac{2}{17325} h q \partial_{x} q\right]+O\left(\epsilon^{2}\right) .
\end{aligned}
$$



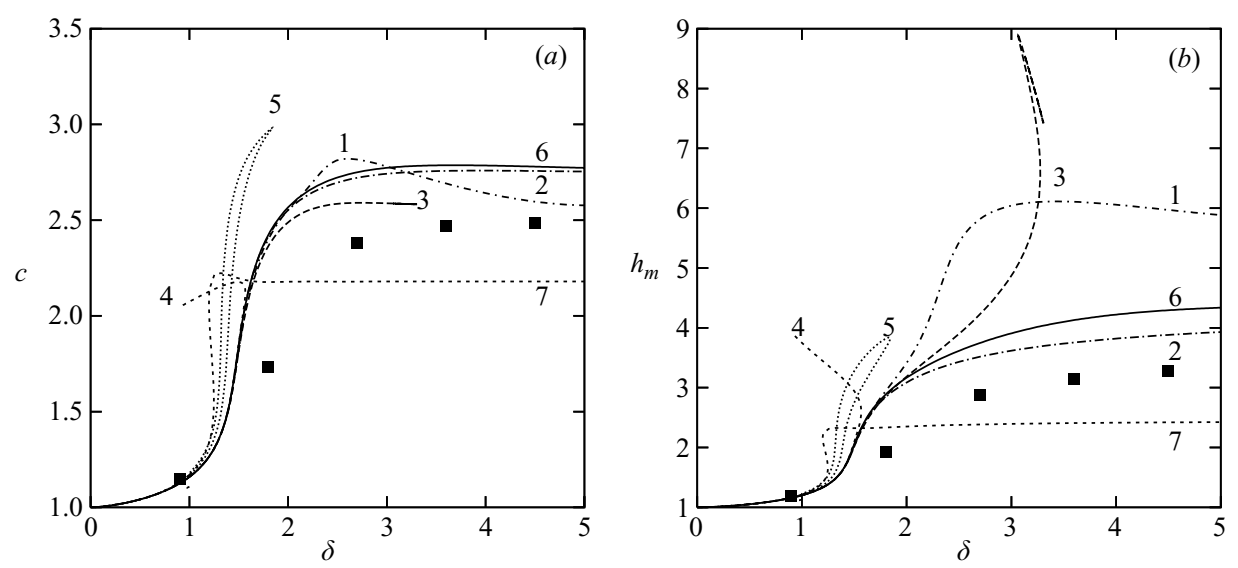

FIGURE 1. (a) Speed $c$ and $(b)$ amplitude $h_{\mathrm{m}}$ of the principal homoclinic orbits as functions of the reduced Reynolds number $\delta$. The wall is vertical and streamwise viscous dissipation is omitted $(\zeta=\eta=0)$. Curve 1 : complete second-order model (3.1), (C 1);2: simplified model (3.1), (4.1); 3 : (3.1), (4.4) with $\mathscr{K}$ given by (4.5); 4 : with $\mathscr{K}$ given by (4.7); 5 : with $\mathscr{K}$ given by (4.8); 6: regularized model (3.1), (4.15); 7: PMB model (3.1), (3.6); filled squares: solutions to the first-order boundary-layer equations after Chang et al. (1996).

Substitution of (4.3) into $\mathscr{R}_{0}$ finally gives

$$
\begin{aligned}
\delta \partial_{t} q & =\frac{5}{6} h-\frac{5}{2} \frac{q}{h^{2}}+\delta\left[\frac{9}{7} \frac{q^{2}}{h^{2}} \partial_{x} h-\frac{17}{7} \frac{q}{h} \partial_{x} q\right]+\delta^{2} \mathscr{K}(h, q) \\
+ & \eta\left[4 \frac{q}{h^{2}}\left(\partial_{x} h\right)^{2}-\frac{9}{2 h} \partial_{x} q \partial_{x} h-6 \frac{q}{h} \partial_{x x} h+\frac{9}{2} \partial_{x x} q\right]-\frac{5}{6} \zeta h \partial_{x} h+\frac{5}{6} h \partial_{x x x} h,
\end{aligned}
$$

where the additional terms arising from the elimination of $r$ and $s$ are second-order inertia terms all collected in $\mathscr{K}$ :

$$
\begin{aligned}
\mathscr{K}= & \frac{1}{210} h^{2} \partial_{t t} q-\frac{1}{105} q \partial_{x} h \partial_{t} q+\frac{1}{42} h \partial_{x} q \partial_{t} q+\frac{17}{630} h q \partial_{x t} q+\frac{653}{8085} q\left(\partial_{x} q\right)^{2} \\
& -\frac{78}{2695} \frac{q^{3}}{h} \partial_{x x} h-\frac{26}{231} \frac{q^{2}}{h} \partial_{x} h \partial_{x} q+\frac{386}{8085} q^{2} \partial_{x x} q+\frac{104}{2695} \frac{q^{3}}{h^{2}}\left(\partial_{x} h\right)^{2} .
\end{aligned}
$$

\subsection{Effective inertial correction terms}

Obviously, these corrections are highly nonlinear. They also contain time derivatives that are difficult to handle, at least in numerical simulations. Fortunately, the zerothorder relation between $q$ and $h$

$$
q=\frac{1}{3} h^{3}
$$

allows us to simplify the expression for $\mathscr{K}$. Using also $\partial_{t} h=-h^{2} \partial_{x} h+O\left(\epsilon^{2}\right)$, we obtain the more compact expression

$$
\mathscr{K}=-\frac{1}{630} h^{7}\left(\partial_{x} h\right)^{2} .
$$

The behaviour of the solutions to equation (4.4) where the inertia corrections $\mathscr{K}$ are given by (4.5) or (4.7) have been tested in the drag-inertia regime by computing the one-hump solitary-wave solutions for a vertical wall and neglecting second-order viscous effects $(\eta=0)$ as explained at the beginning of $\S 5$. Figure 1 displays the speed and amplitude of the solitary waves as a function of the reduced Reynolds number $\delta$. They are compared to the solutions to the complete second-order model (3.1), (C 1) 
as curves 1, to the simplified model (3.1), (4.1) as curves 2, to the PMB model (3.1), (3.6) as curves 7, and to the results obtained by Chang, Demekhin \& Kalaidin (1996) with the first-order boundary-layer equations as filled squares.

The simplified model and the complete second-order model both exhibit unique one-hump solitary-wave solutions at given $\delta$ and have speed in reasonable agreement with the results of Chang et al. On the contrary, the branch of principal homoclinic solutions is seen to turn back in the transition region between the drag-gravity and the drag-inertia regimes $(\delta \sim 1)$ with both expressions $(4.5)$ and (4.7) for $\mathscr{K}$ (curves 3 and 4 in figure 1). This unphysical behaviour is similar to the one encountered with the Benney equation (3.3) and is likely to be related to the high-degree nonlinearities present in (4.5) and (4.7). The same difficulty as in the case of surface equations arises and calls for a formulation in which inertia effects are accurately accounted for in the widest possible range of reduced Reynolds numbers $\delta$.

Other forms of the second-order inertia corrections $\mathscr{K}$ can be obtained by using the flat-film relation (4.6). For example, Roberts (1996) has applied a centre manifold analysis to the problem of a falling film and derived a second-order model in terms of the film thickness $h$ and the depth-averaged velocity equivalent to the flow rate $q$. His approach relied on the linear viscous dissipating modes of the streamwiseuniform film in the zero-wavenumber limit, which is basically a reduction of the slow time and space evolution of the film to the two first eigenmodes $(h, u) \propto(1,0)$ and $(h, u) \propto(0, \sin (\pi y /(2 h)))$. His model is similar to those obtained using the classical depth-averaged method with coefficients close to those appearing in (4.4). As noticed by Ooshida (1999), this agreement can be understood from the fact that the velocity profile $u_{\text {rob }} \propto \sin (\pi y /(2 h))$ is very close to the parabolic profile since $\left\langle u_{\text {rob }}, g_{0}\right\rangle / \sqrt{\left\langle u_{\text {rob }}, u_{\text {rob }}\right\rangle\left\langle g_{0}, g_{0}\right\rangle} \approx 0.999$. Inertia corrections obtained by Roberts are

$$
\begin{aligned}
\mathscr{K}= & \frac{1}{100}\left(-0.1961 \frac{q^{3}}{h^{2}}\left(\partial_{x} h\right)^{2}-1.78 \frac{q^{2}}{h} \partial_{x} h \partial_{x} q+0.1226 q\left(\partial_{x} q\right)^{2}\right. \\
& \left.-1.792 \frac{q^{3}}{h} \partial_{x x} h+0.7778 q^{2} \partial_{x x} q\right) .
\end{aligned}
$$

The results obtained with this expression for $\mathscr{K}$ are also displayed in figure 1 as curves 5 . A loss of solutions is once more observed at $\delta \approx 2$, a failure due to the fact that $\mathscr{K}$ is obtained from a perturbation method which is strictly valid only in the drag-gravity regime where inertia has a perturbative role only. Our derivations of (4.4) with $\mathscr{K}$ given by (4.5) or (4.7) are also based on perturbative techniques applied to the Nusselt flat-film solution. However, the presence of the principal homoclinic solutions to the simplified model (3.1), (4.1) for all $\delta$ shows that it should be possible to describe the drag-inertia regime at low cost in terms of a model including the second-order inertial effects and involving $h$ and $q$ only.

\subsection{Padé-like regularization}

Here, we follow a procedure more closely inspired by the Padé approximant technique than Ooshida's, by looking for a kind of algebraic preconditioner able to remove the dangerous second-order terms of inertia origin (in $\delta^{2}$ ). Instead of thinking in terms of an expansion of the flow rate $q$, we consider the residual $\mathscr{R}_{0}$ obtained by averaging the momentum equation (2.8) with weight $g_{0}$, which can be written as a series in $\epsilon, \mathscr{R}_{0}^{(0)}+\mathscr{R}_{0}^{(1)}+\mathscr{R}_{0}^{(2), \eta}+\mathscr{R}_{0}^{(2), \delta}$. In the second-order terms of this expansion, we have isolated those having a viscous origin (superscript $\eta$ ) from those accounting for the convective acceleration induced by the deviations of the velocity profile from the 
parabolic shape (superscript $\delta$ ). The simplified equation (4.1) is recovered just by neglecting $\mathscr{R}_{0}^{(2), \delta}$. So $\mathscr{R}_{0}$ is sought in the form $\mathscr{G}^{-1} \mathscr{F}$ where $\mathscr{G}$ is now simply a function of $h, q$ and their derivatives, and $\mathscr{F}$ is reduced to $\mathscr{R}_{0}^{(0)}+\mathscr{R}_{0}^{(1)}+\mathscr{R}_{0}^{(2), \eta}$, i.e. the residual that was obtained assuming a parabolic velocity profile. Setting $\mathscr{F}=\mathscr{G} \mathscr{R}_{0}$ to zero gives

$$
\begin{aligned}
& \delta \mathscr{G}(h, q) \int_{0}^{h} g_{0}(y / h)\left[\partial_{t} u+u \partial_{x} u+v \partial_{y} u\right] \mathrm{d} y \\
& \quad=\mathscr{G}(h, q) \int_{0}^{h} g_{0}(y / h)\left\{1+\partial_{y y} u-\zeta \partial_{x} h+\partial_{x x x} h+\eta\left(2 \partial_{x x} u-\partial_{x}\left[\left.\partial_{y} v\right|_{h}\right]\right)\right\} \mathrm{d} y,
\end{aligned}
$$

where inertia terms isolated on the left-hand side are

$$
\begin{aligned}
\delta \mathscr{G} & \int_{0}^{h} g_{0}(y / h)\left[\partial_{t} u+u \partial_{x} u+v \partial_{y} u\right] \mathrm{d} y \\
= & \delta \mathscr{G}\left\{\left[\frac{2}{5} \partial_{t} q-\frac{18}{35} \frac{q^{2}}{h^{2}} \partial_{x} h+\frac{34}{35} \frac{q}{h} \partial_{x} q\right]-\frac{2}{5} \delta \mathscr{K}\right\} \equiv \mathscr{G}\left\{\mathscr{R}_{0}^{(1), \delta}+\mathscr{R}_{0}^{(2), \delta}\right\},
\end{aligned}
$$

which we want to identify with

$$
\delta\left[\frac{2}{5} \partial_{t} q-\frac{18}{35} \frac{q^{2}}{h^{2}} \partial_{x} h+\frac{34}{35} \frac{q}{h} \partial_{x} q\right] \equiv \mathscr{R}_{0}^{(1), \delta} .
$$

This leads to taking the regularization factor as

$$
\mathscr{G}=\left[1+\frac{\mathscr{R}_{0}^{(2), \delta}}{\mathscr{R}_{0}^{(1), \delta}}\right]^{-1}
$$

An asymptotically equivalent expression for $\mathscr{G}$ can be found using $q=h^{3} / 3+O(\epsilon)$, and $\partial_{t} h=-h^{2} \partial_{x} h+O\left(\epsilon^{2}\right)$. We then obtain

$$
\mathscr{R}_{0}^{(1), \delta}=-\frac{2}{15} \delta h^{4} \partial_{x} h+O\left(\epsilon^{2}\right) \quad \text { and } \quad \mathscr{R}_{0}^{(2), \delta}=\frac{\delta^{2}}{1575} h^{7}\left(\partial_{x} h\right)^{2}+O\left(\epsilon^{3}\right),
$$

which, when substituted in (4.12), yields

$$
\mathscr{G}=\left[1-\frac{\delta}{210} h^{3} \partial_{x} h\right]^{-1}+O\left(\epsilon^{2}\right)
$$

In order to lower the degree of nonlinearity as much as possible, $\mathscr{G}$ is finally rewritten in terms of the local slope $\partial_{x} h$ and the local Reynolds number $\delta q$ :

$$
\mathscr{G}=\left[1-\frac{\delta}{70} q \partial_{x} h\right]^{-1}
$$

The resulting set of equations is

$$
\begin{aligned}
\delta \partial_{t} q= & \delta\left[\frac{9}{7} \frac{q^{2}}{h^{2}} \partial_{x} h-\frac{17}{7} \frac{q}{h} \partial_{x} q\right] \\
& +\left\{\frac{5}{6} h-\frac{5}{2} \frac{q}{h^{2}}+\eta\left[4 \frac{q}{h^{2}}\left(\partial_{x} h\right)^{2}-\frac{9}{2 h} \partial_{x} q \partial_{x} h-6 \frac{q}{h} \partial_{x x} h+\frac{9}{2} \partial_{x x} q\right]\right. \\
& \left.-\frac{5}{6} \zeta h \partial_{x} h+\frac{5}{6} h \partial_{x x x} h\right\}\left[1-\frac{\delta}{70} q \partial_{x} h\right]^{-1},
\end{aligned}
$$

along with the mass balance equation (3.1). 
Hereafter, the system (3.1), (4.15) will be referred to as the regularized model. Homoclinic orbits corresponding to one-hump solitary-wave solutions to (3.1), (4.15) have been computed and are displayed as curves 6 in figure 1. Non-physical turning back of the curves has never been observed for all the values of $\delta$ studied. Moreover, system (3.1), (4.15) is fully consistent at second order with the Benney expansion and takes into account modifications of the momentum balance of the film induced by the deviations of the velocity profile from the parabolic Nusselt solution.

\section{Validation}

Periodic waves at rest in their moving frame $\xi=x-c t$, where $c$ is the wave speed, have been computed by continuation using AuT097, which, together with its package HomConT (Doedel et al. 1997) has been used extensively to obtain numerical results. Their characteristics are then compared to available DNS results (Salamon, Armstrong \& Brown 1994; Ramaswamy, Chippada \& Joo 1996; Malamataris, Vlachogiannis \& Bontozoglou 2002) and laboratory experiments (Liu \& Gollub 1994). For stationary waves, the mass conservation equation (3.1) can be integrated once to give

$$
q=c h+q_{0},
$$

where $q_{0}$ is an integration constant corresponding to the conservation of the flow rate in the moving frame. Denoting by $\langle\cdot\rangle=\Lambda^{-1} \int_{0}^{\Lambda}(\cdot) \mathrm{d} \xi$ the average operator over a wavelength $\Lambda$ in the moving frame, the constant $q_{0}$ is adjusted at every step of the continuation procedure to ensure either $\langle h\rangle=1$ or $\langle q\rangle=1 / 3$. The constant-thickness formulation corresponds to the periodic-boundary conditions often used in numerical simulations, whereas the constant-flux formulation is encountered when the spatial development of a time-periodic signal is considered (Scheid et al. 2005b).

\subsection{Comparisons to direct numerical simulations}

Salamon et al. (1994) have performed a systematic analysis of the two travelling-wave branches of slow $\gamma_{1}$ and fast $\gamma_{2}$ solutions observed experimentally. Their numerical scheme enforces a constant averaged thickness $\langle h\rangle=1$. In figure 2 , we reproduce the bifurcation diagrams in the parameter space (speed $c$, wavenumber $k$ ) for the largest value of the reduced Reynolds number $\delta$ tested by these authors, $\delta=2.79$ (Salamon et al. 1994, figures 15 and 17). For weak viscous diffusion $\eta=0.015$, the $\gamma_{1}$ family emerges from a Hopf bifurcation of the Nusselt flat-film solution at the marginal wavenumber $k_{c} \approx 1.02$, whereas the $\gamma_{2}$ family bifurcates from the $\gamma_{1}$ branch by period doubling at a wavenumber close to $k_{c} / 2$. At a larger viscous diffusion $\eta=0.075$, the situation is reversed. Computations with our regularized model (3.1), (4.15) are compared to findings by Salamon et al. in figure 2, showing excellent agreement. Wave profiles and streamlines in the moving frame of reference are also displayed. As can be observed from the DNS by Salamon et al. the capillary oscillations following the $\gamma_{1}$ waves or preceding the $\gamma_{2}$ waves are damped by viscous diffusion (Salamon et al. 1994, figures 16 and 18).

Travelling waves obtained by DNS when a periodical forcing is simulated at inlet correspond to the constant-flux formulation $\langle q\rangle=1 / 3$. Our results are displayed in figure 3 together with the corresponding numerical solution obtained by Malamataris et al. (2002) and the results from the PMB model. Parameters are those of the experiment by Liu \& Gollub (1994) with $\beta=6.4^{\circ}, R=19.33, \Gamma=526$, and forcing 
(i) (a)

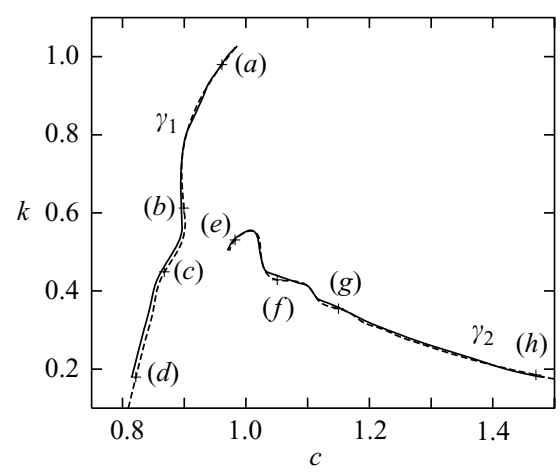

(ii) (a)

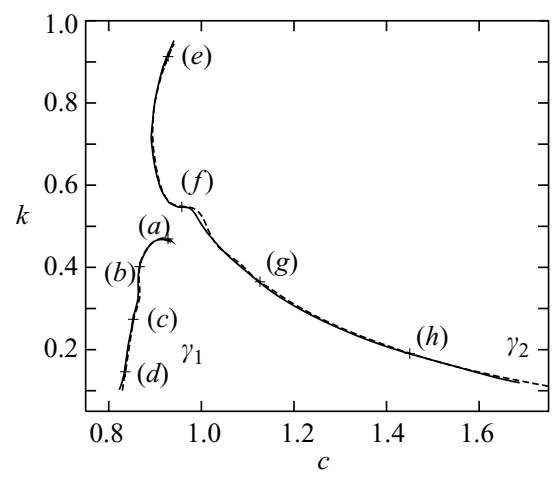

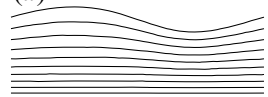

(b)

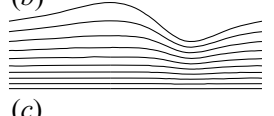

(c)

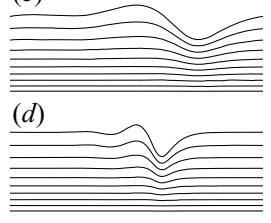

(e)
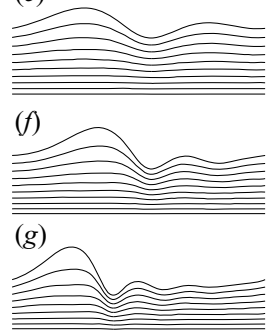

(h)

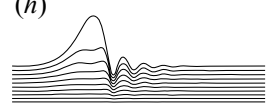

(e)

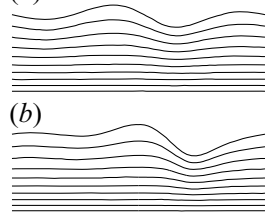

(c)

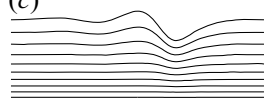

(d)

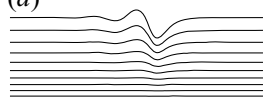

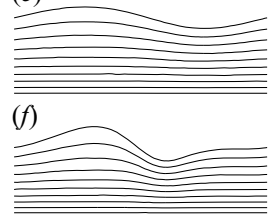

(g)

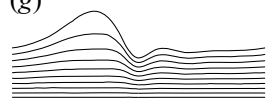

(h)

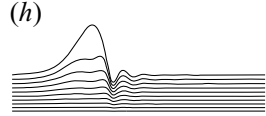

FIGURE 2. Travelling-wave families $\gamma_{1}$ and $\gamma_{2}$. Dashed lines refer to solutions to the regularized model (3.1), (4.15), whereas solid lines refer to the results from DNS (after Salamon et al. 1994). Left: speed $c$ as function of the wavenumber $k$; right: wave profiles and streamlines in the moving frame of reference for wave families $\gamma_{1}$ (labels $a$ to $d$ ) and $\gamma_{2}$ (labels $e$ to h). (i) $\delta=2.79, \zeta=0$ and $\eta=0.015(R=7.59$ and $\Gamma=4374)$; (ii) $\delta=2.79, \zeta=0$ and $\eta=0.075$ $(R=3.40$ and $\Gamma=228.8)$.

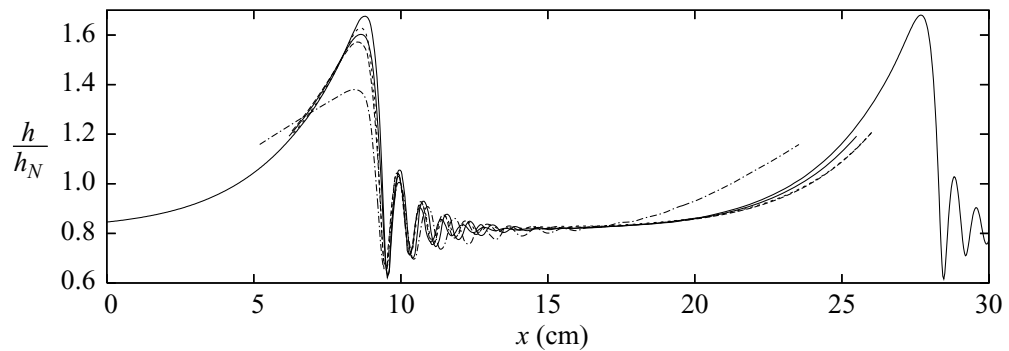

FIGURE 3. Travelling wave train approaching solitary waves corresponding to an experiment by Liu \& Gollub (1994). The forcing frequency is $f=1.5 \mathrm{~Hz}$. Parameters are $\beta=6.4^{\circ}, R=19.33$ and $\Gamma=526(\delta=17.7, \zeta=2.72$ and $\eta=0.093)$. The thin solid line shows the numerical computation by Malamataris et al. (2002). Results of the regularized (simplified) model correspond to the thick solid (dashed) line. The solution to the complete second-order model is the dotted line and the solution to the PMB model is the dashed-dotted line. 
frequency $f=1.5 \mathrm{~Hz}$. Results of our models are in excellent agreement with the DNS results, predicting a wave amplitude that is slightly smaller than the one given by the Navier-Stokes equations. The complete model gives the closest answer whereas the amplitude of the solution to the simplified model is significantly lower. As could be expected, the prediction of the regularized model lies somewhere in between. Regarding wavelengths, the regularized model gives a value closer to that given by Malamataris' computation than the simplified and the complete second-order models. Sharing the main characteristics of the solitary waves obtained by Ooshida, unsurprisingly the solutions to the PMB model (3.1), (3.6) capture their properties much less faithfully than those obtained from our regularization procedure.

Malamataris et al. (2002) questioned the similarity assumption of a parabolic velocity profile. They showed that significant differences could be found in the case of large solitary waves. Deviations from the parabolic profile were mostly located in front of the main hump and behind the first capillary ripple, where gradients of the film thickness are the largest. They also proved the occurrence of a counterflow $(u<0)$ in the region bracketing the thickness minimum. This feature, confirmed in the experiments by Tihon et al. (2003), is reproduced well in our models. We have reconstructed the streamwise velocity distributions based on expansions truncated beyond the three first polynomials $g_{0}, g_{1}$ and $g_{2}$ (see Appendix A for details) at regularly spaced locations around that minimum and for the wave train shown in figure 3. Corresponding profiles are displayed in figure 4 for the complete secondorder model $(b, d)$ and the regularized model $(a, c)$. The presence of a counterflow is recovered in both cases. The similarity with DNS results is particularly convincing for the complete second-order model both at behind the first ripple and in front of the main hump (figure 4 compared to figure 7 in Malamataris et al. (2002); even the two inflection points they observed are recovered). For the regularized model, comparisons remain satisfactory everywhere except at the front of the main hump where the gradients are the largest. This implies that a reconstruction of the velocity field based on the expressions at first order for $r$ and $s$ given by (4.3) is no longer sufficient there, whereas the agreement is re-obtained once the slope of the interface is less steep.

The streamlines in the moving frame of reference, i.e. the isocontours of the streamfunction $\int_{0}^{y}(u-c) d y$, show little difference whether the complete or the regularized model is considered (the streamlines computed with the regularized model are shown in figure $4 e$ ). The reason is that, at the thickness minimum where the relative deviations away from the parabolic profile are noticeable, the streamwise velocity $u$ is also small, so that in a reference frame moving at the speed of the wave, the velocity $u-c$ remains everywhere close to that corresponding to a parabolic profile.

\subsection{Comparisons to experiments}

To complete the validation of our models, we present results for the speeds and amplitudes of solitary waves corresponding to experimental conditions considered by Liu \& Gollub (1994) for an inclined plane at a fixed angle $\beta=8^{\circ}$ using a glycerin-water mixture $(\Gamma=488)$, and at different Reynolds numbers. The experimental findings are compared to the corresponding travelling-wave results in figure 5. Solutions to (4.1), (4.15), and (C 1) completed with (3.1) were obtained by varying the wavelength and imposing a constant average flow rate $\langle q\rangle=1 / 3$. In order to obtain the $\gamma_{2}$ branch, we proceeded by starting from the linear threshold for a vertical film and high viscous second-order diffusion $\eta$ (or low $\Gamma$ ) since it is known that the $\gamma_{2}$ waves then emerge 

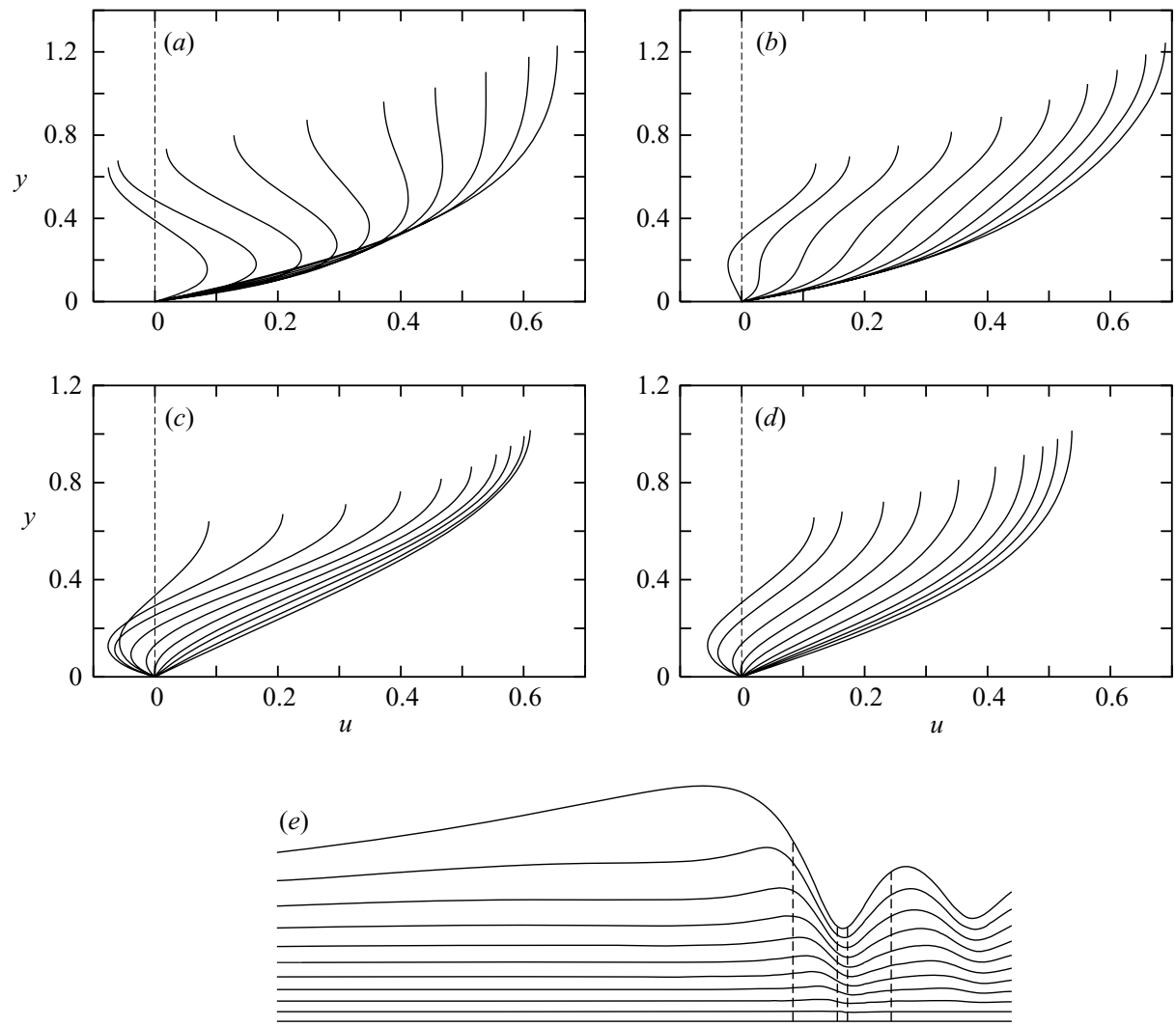

(a) (c)

FIGURE 4. Streamwise velocity profile at regularly spaced locations from the front of the solitary hump $(a, b)$ to the back of the first capillary ripple $(c, d)$. $(a, c)$ Solutions to the regularized model $(3.1),(4.15) ;(b, d)$ solutions to the complete second-order model $(3.1),(\mathrm{C} 1) ;(e)$ wave profile and streamlines corresponding to the regularized model. Extremal positions of the given velocity profiles are indicated by dashed lines.

from the flat-film solution through a Hopf bifurcation. We next adjusted the slope $(\beta)$ and surface tension $(\Gamma)$ to the desired values. The $\gamma_{2}$ branch of solutions is found to extend from high speed to low speed, where it terminates as a negative solitary pulse. The curves globally have a linear shape in agreement with the linear relation between maximum height and wave speed obtained by Chang (1986) for solitary waves through a normal form analysis of the Kuramoto-Sivasinsky equation. Both the regularized model and the complete second-order model predict maximum heights larger than the experimental findings, which is in agreement with the DNS of the primitive equations that also predicted larger amplitudes than the experimental wave profiles observed by Liu \& Gollub, see Ramaswamy et al. (1996); Malamataris et al. (2002). This peculiarity could be a consequence of the neglect of the transverse curvature of the humps, or else a slight smoothing of the wave crests linked to the experimental measurement of the thickness. The near-perfect agreement between experiments and the results from the simplified model (4.1) is therefore accidental. For comparison, the results from the PMB model (3.1), (3.6) are also reported in figure 5(a) showing again less convincing agreement than with our models. 

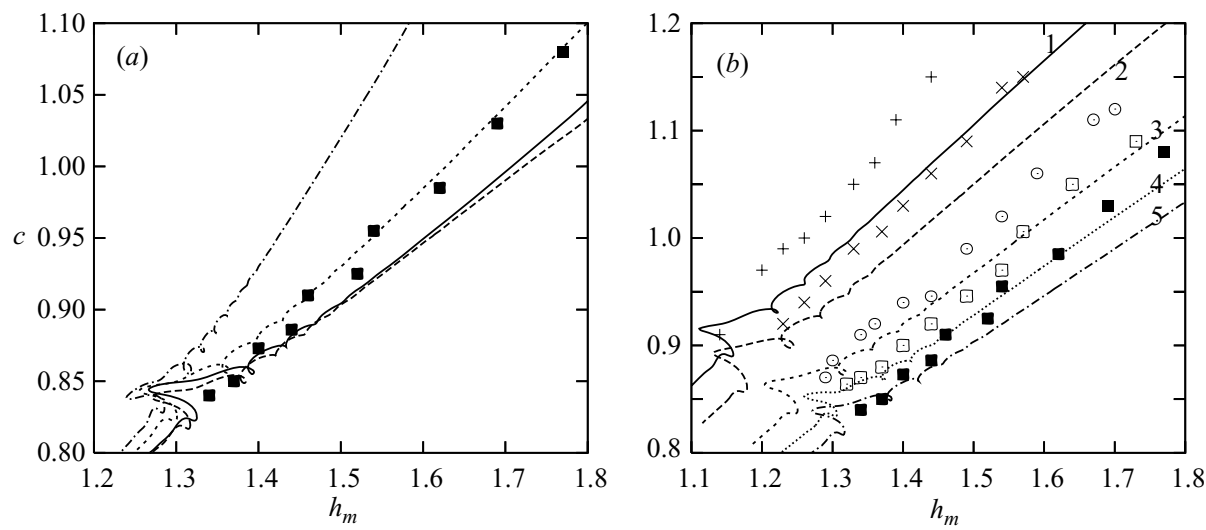

FIGURE 5. Phase speed of solitary-like wave trains as a function of their peak height compared to the experimental data provided by Liu \& Gollub (1994) (shown as symbols) at $\beta=8^{\circ}$, $\Gamma=488$. (a) $R=20.1$, comparison to the corresponding travelling wave families computed with the complete second-order model (3.1), (C1) (solid line), the regularized model (3.1), (4.15) (dashed line), the simplified model (3.1), (4.1) (dotted line) and the PMB model (3.1), (3.6) (dashed-dotted line); (b) comparison to the solutions of the regularized model for different Reynolds numbers, $R=11.9(1,+), 13.6(2, \times), 16.8(3, \odot), 18.7(4, \square)$ and $20.1(5, \mathbf{\square})$.

From the above comparisons, it can be concluded with some confidence that our models predict correctly the shape, speed and maximum height of the solitary waves at least in the range of parameters explored by Liu \& Gollub.

\section{Two-dimensional modelling of three-dimensional film flows}

We now turn to the three-dimensional formulation of the problem, and look for two-dimensional equations in the streamwise $(x)$ and spanwise $(z)$ coordinates that mimic the full three-dimensional motion of the fluid. The flat-film solution is a parallel flow with no spanwise component, i.e. $w=0$. A valid approach is therefore to consider $w$ of order $\epsilon$, with the meaning that spanwise flows are triggered by the modulations of the free surface. Ruyer-Quil \& Manneville (2000) used this assumption to simplify the cumbersome system of equations which models the three-dimensional flow dynamics. However, considering the continuity equation (2.2), the least-degeneracy principle suggests $w$ be taken as an $O(1)$ quantity like $u$ and this is the approach we will take below.

Following the same procedure as for the two-dimensional case, one finds that six fields are needed to account for the velocity components at second order: $q, r, s$, the spanwise flow rate $q_{z}=\int_{0}^{h} w \mathrm{~d} y$, and the corrections $r_{z}$ and $s_{z}$. For symmetry, the streamwise flow rate and the corrections to the streamwise parabolic velocity profile are hereafter denoted by $q_{x} \equiv q, r_{x} \equiv r$ and $s_{x} \equiv s$. The boundary-layer equations are then averaged using the Galerkin method by writing residuals $\left\langle E_{\alpha}, g_{i}\right\rangle$ where $\langle f, g\rangle=\int_{0}^{h} f g \mathrm{~d} y$, while $\alpha=x$ and $\alpha=z$ refer to the streamwise and spanwise momentum balances, respectively. These residuals yield a system of six evolution equations for $h, q_{\alpha}, r_{\alpha}$ and $s_{\alpha}$ completed with the mass balance obtained through integration of (2.2) across the layer depth $\partial_{t} h+\partial_{x} q_{x}+\partial_{z} q_{z}=0$. The full expression of the complete model is very cumbersome and is provided in Appendix $\mathrm{C}$ as equations (C1). First-order expressions for the four fields $r_{\alpha}, s_{\alpha}$ are readily obtained from the truncation at order $\epsilon$ of the residuals corresponding to the weights $g_{1}$ and 
$g_{2}$. Substitution of these expressions in the first two residuals $\mathscr{R}_{0, \alpha}=\left\langle E_{\alpha}, g_{0}\right\rangle$ produces second-order inertia terms, formally written as $\mathscr{R}_{0, \alpha}^{(2), \delta}$. These terms contain highorder nonlinearities that we next eliminate by adjusting algebraic preconditioners. So residuals $\mathscr{R}_{0, \alpha}$ are sought in the form $\mathscr{G}_{\alpha}^{-1} \mathscr{F}_{\alpha}$ where the $\mathscr{F}_{\alpha}$ correspond to the expressions for the residuals $\mathscr{R}_{0, \alpha}$ when a parabolic velocity profile is assumed, i.e. when corrections $r_{\alpha}$ and $s_{\alpha}$ are neglected. Isolating inertia terms, we thus set

$$
\mathscr{G}_{\alpha}\left(\mathscr{R}_{0, \alpha}^{(1), \delta}+\mathscr{R}_{0, \alpha}^{(2), \delta}\right)=\mathscr{R}_{0, \alpha}^{(1), \delta},
$$

where superscripts refer to first-order and second-order inertia terms. Zeroth-order expressions for the flow rates $q_{x}=h^{3} / 3+O(\epsilon)$ and $q_{z}=O(\epsilon)$, i.e. the gravity-oriented (base) flow, are next invoked to reduce the degree of nonlinearity of the regularization factors $\mathscr{G}_{\alpha}$. Consequently, the inertia terms $\mathscr{R}_{0, z}^{(2), \delta}$ induced by deviations of the spanwise velocity field from the parabolic profile appear asymptotically at order $\epsilon^{3}$. So that we merely obtain $\mathscr{G}_{z}=1+O\left(\epsilon^{2}\right)$. Similarly, the asymptotic expression for $\mathscr{R}_{0, x}^{(2), \delta}$ corresponds exactly to the one obtained for a spanwise-independent flow. Hence we have $\mathscr{G}_{z} \equiv 1$ and $\mathscr{G}_{x} \equiv \mathscr{G}$ given in (4.14). The three-dimensional extension of the regularized model, whose explicit expression is given in Appendix B, is formally written:

$$
\begin{aligned}
\partial_{t} h & =-\partial_{x} q_{x}-\partial_{z} q_{z}, \\
\delta \partial_{t} q_{x} & =\delta \mathscr{I}_{x}^{2 D}+\mathscr{G}_{x}\left\{\frac{5}{6} h-\frac{5}{2} \frac{q_{x}}{h^{2}}+\delta \mathscr{I}_{x}^{3 D}+\eta\left[\mathscr{D}_{x}^{2 D}+\mathscr{D}_{x}^{3 D}\right]+\frac{5}{6} h \partial_{x} \mathscr{P}\right\}, \\
\delta \partial_{t} q_{z} & =\delta \mathscr{I}_{z}^{2 D}-\frac{5}{2} \frac{q_{z}}{h^{2}}+\delta \mathscr{I}_{z}^{3 D}+\eta\left[\mathscr{D}_{z}^{2 D}+\mathscr{D}_{z}^{3 D}\right]+\frac{5}{6} h \partial_{z} \mathscr{P},
\end{aligned}
$$

where $\mathscr{I}$ and $\mathscr{D}$ stand for terms of inertia and viscous diffusion origin, easily identified in (B $1 b)$ and (B 1c). The two terms $\mathscr{P}=-\zeta h+\left(\partial_{x x}+\partial_{z z}\right) h$ stem from the gravitational and capillary contributions to the pressure respectively. In (6.2b), we have also isolated terms already present in the two-dimensional model (superscript $2 D$ ) from those arising from the spanwise dependence (superscript $3 D$ ). Expressions collected into terms with subscripts $x$ are obtained from those with subscripts $x$ by making the exchanges $\{x \leftrightarrow z\}$.

Equations $(6.2 a),(6.2 b),(6.2 c)$ express mass conservation, and the averaged momentum balance in directions $x$ and $z$, respectively. The viscous drag corresponds to the terms $\frac{5}{2} q_{x} / h^{2}$ in $(6.2 b)$ and $\frac{5}{2} q_{z} / h^{2}$ in $(6.2 c)$. In the boundary-layer equations, gravity contributes only to the streamwise momentum balance through the term $\frac{5}{6} h$ in $(6.2 b)$.

The regularized model (6.2) is fully consistent with the Benney expansion at second order, whereas the three-dimensional simplified model, corresponding to the averaging of the momentum balance equations across the layer depth assuming both parabolic velocity profiles and weights, is not. The latter can be recovered from the former by replacing the factor $\mathscr{G}_{x}$ by $1 . \dagger$

$\dagger$ Notice that the simplified model formulated by Ruyer-Quil \& Manneville (2000) contains two typing mistakes in their equation (54): terms $-\frac{97}{56} q_{x} \partial_{z} q_{z} / h$ and $\frac{129}{56} q_{x} q_{z} \partial_{z} h / h^{2}$ should be corrected to read $-\frac{8}{7} q_{x} \partial_{z} q_{z} / h$ and $\frac{9}{7} q_{x} q_{z} \partial_{z} h / h^{2}$. 


\section{Floquet analysis}

In this section, the stability of two-dimensional waves to transverse perturbations is investigated in the experimental conditions considered by Liu et al. (1995). Experimental profiles indicate that the waves selected by the linear inception are of type $\gamma_{1}$, slow waves with deep troughs and bumped crests. Our efforts have accordingly been concentrated on the stability analysis of the $\gamma_{1}$ travelling waves. These waves were computed using Auto97 (Doedel et al. 1997) by continuation. The constant flux condition $\left\langle q_{x}\right\rangle=1 / 3$ was enforced (see $\S 5$ ). We started from infinitesimal sinusoidal waves at linear threshold, and increased the period. A standard Floquet stability analysis of the wave to both streamwise and spanwise modulations was performed next. Each unknown field $X$ in the frame moving with the wave $\xi=x-c t$ was expressed as $X(\xi, z, t)=X_{0}(\xi)+\varepsilon X_{1}(\xi, z, t)$ where $\varepsilon \ll 1$ and $X_{0}$ is the basic two-dimensional travelling wave. The perturbation $X_{1}$ was expanded as $\sum_{\varphi_{i} k_{z}} \tilde{X}_{\varphi, k_{z}}(\xi) \exp \left\{\mathrm{i} \varphi k_{x} \xi+\mathrm{i} k_{z} z+s t\right\}$ where $\tilde{X}_{\varphi, k_{z}}$ is periodic in $\xi$ with period $2 \pi / k_{x}$, $k_{x}$ is the wavenumber of the two-dimensional basic stationary wave, and $k_{z}$ is the wavenumber of the transverse perturbation. The detuning parameter, $\varphi$, is the ratio of the streamwise wavenumber of the perturbation to that of the base state, hence $\varphi \in[0,1[. \varphi \in \mathbb{Q}$ signals a subharmonic mode, especially $\varphi=1 / 2$, and $\varphi \notin \mathbb{Q}$ an incommensurate modulated mode. Denoting $\boldsymbol{X}_{0}(\xi)$ the vector formed by the different components of the base state, and $\tilde{\boldsymbol{X}}$ the vector formed by the amplitudes of the perturbations, the linearized set of equations can be formally written as

$$
\varsigma \tilde{\boldsymbol{X}}=\mathscr{L}\left(\boldsymbol{X}_{0} ; c, q_{0}, \delta, \zeta, \eta, \varphi, k_{z}\right) \tilde{\boldsymbol{X}}
$$

where $\mathscr{L}$ is a linear operator and $\varsigma$ is the complex growth rate. The maximum real part denoted $\zeta_{r}^{M}$ corresponds to the fastest growing perturbation of interest from the experimental point of view. The parameter space $\varphi \times k_{z}$ can be reduced by invoking two symmetries: (i) reflection in the spanwise direction, which allows us to consider only positive $k_{z}$; (ii) invariance of (7.1) under the transformation $\left(\varphi, k_{z}, \varsigma, \tilde{\boldsymbol{X}}\right) \rightarrow$ $\left(-\varphi,-k_{z}, \varsigma^{\star}, \tilde{\boldsymbol{X}}^{\star}\right)$, the star denoting complex conjugation. The parameter space $\varphi \times k_{z}$ can thus be limited to $\left[0, \frac{1}{2}\right] \times[0, \infty[$. The eigenvalue problem (7.1) was solved in Fourier space where 256 real modes have been used to represent the computed twodimensional waves and 128 complex modes to represent the perturbation (limited to 32 for the complete model owing to its complexity). Eigenvalues and eigenvectors were computed using a QR algorithm implemented on an RS/6000 IBM workstation.

Liu et al. (1995) considered a falling film of a glycerol-water mixture $\left(\rho=1070 \mathrm{~kg} \mathrm{~m}^{-3}, \nu=2.3 \times 10^{-6} \mathrm{~m}^{2} \mathrm{~s}^{-1}\right.$ and $\left.\sigma=67 \times 10^{-3} \mathrm{~N} \mathrm{~m}^{-1}\right)$, with $\beta=6.4^{\circ}$ and $R=56$. They measured the wavelength of the two-dimensional base state $\lambda_{x}$ as well as the wavelength of the transverse modulations $\lambda_{z}$, obtained by varying the frequency of the periodic forcing. Results of Floquet analysis using the complete, regularized and simplified models are presented in figure 6 using dimensional units. In line with the results discussed in $\S 5$, the computed wavelengths $\lambda_{x}$ of $\gamma_{1}$ waves are of the same order of magnitude as found experimentally, see figure $6(a)$. Our computations also indicate relatively small variations of $\lambda_{z}$ with the frequency, which is in agreement with the results reported by Liu et al. (see figure $6 b$ ). The transverse wavelengths of the most amplified perturbations for the regularized and the complete models agree with each other, whereas the simplified model predicts larger wavelengths. This shows the role of the second-order inertia terms arising from corrections to the velocity profile in the mechanism of the three-dimensional secondary instability. At low frequency, $\lambda_{z}$ goes to infinity so that the most amplified perturbation is spanwise-uniform, while the 

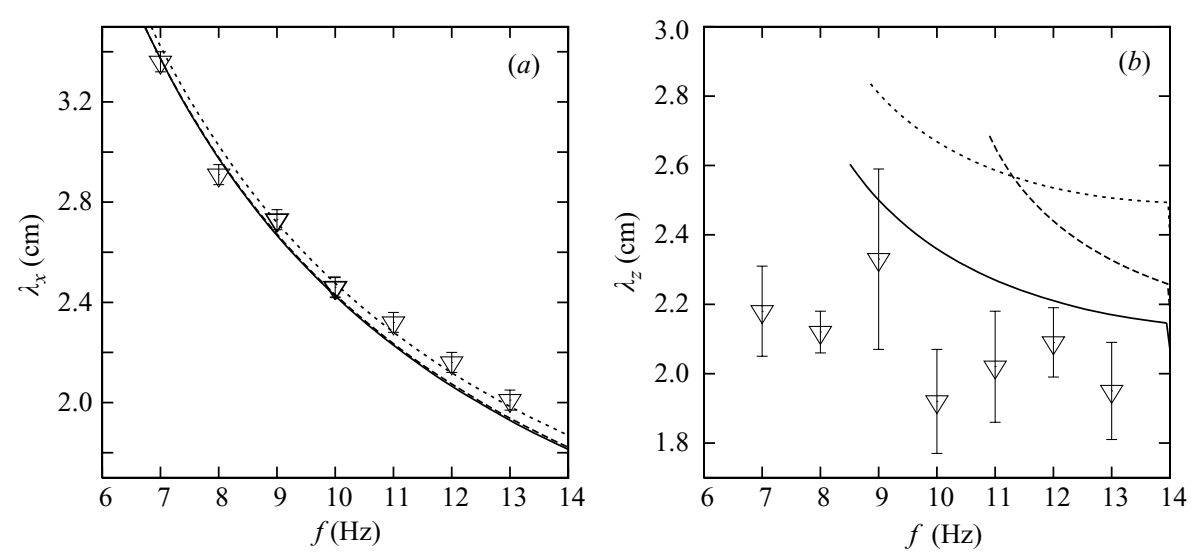

FIGURE 6. (a) Streamwise wavelengths $\lambda_{x}$ of two-dimensional waves and $(b)$ spanwise wavelengths $\lambda_{z}$ of the fastest growing three-dimensional perturbations versus the forcing frequency $f$, with $\beta=6.4^{\circ}, R=56$ and $\Gamma=2002$. Down triangles are experimental findings by Liu et al. (1995). Solid, dashed and dotted lines correspond to the complete model, the regularized model (6.2) and the simplified model, respectively. In panel (a), solid and dashed lines are nearly superimposed.

experimental $\lambda_{z}$ remains finite. Another difference is that Floquet analysis predicts that the detuning parameter for the fastest growing perturbation (not shown) corresponds to a subharmonic secondary instability $\left(\varphi=\frac{1}{2}\right.$, chequerboard pattern) rather than to the synchronous transition $(\varphi \approx 0)$ reported by Liu et al.

Figure $7(a)$ summarizes the experimental findings by Liu et al. in the $(R, f)$ plane for the same glycerol-water mixture and with $\beta=4^{\circ}$. Liu et al. reported two different scenarios that are strongly reminiscent of what happens in boundary layers (Schmid \& Henningson 2001). The first one, referred to as a synchronous mode, is characterized by wave crests deformed in phase in the spanwise direction. The second one, less commonly observed, appears when two successive crests are deformed with a phase shift of $\pi$. This leads to chequerboard (or herringbone) patterns characteristic of a streamwise subharmonic instability combined with a spanwise modulation. These two modes are reminiscent of aligned and staggered $\Lambda$-vortices developing in transitional boundary layers, thus analogous to the type- $\mathrm{K}$ and type- $\mathrm{H}$ transitions, respectively (Herbert 1988). Corresponding results for the stability of $\gamma_{1}$ waves are presented in figure $7(b-d)$, as obtained from the regularized model. The results for the solutions to the complete and simplified models are very similar to those obtained with the regularized model and thus not shown. We have computed the detuning parameter (figure $7 b$ ) and the spanwise wavenumber (figure $7 c, d$ ) of the fastest growing perturbation, with a Reynolds step of 1 and a frequency step of $1 \mathrm{~Hz}$ (the lowest frequency considered is $4 \mathrm{~Hz}$ owing to the large number of modes necessary to represent the solution in that case). Computations show that $k_{z}$ decreases steadily as $R$ is reduced and goes to zero in a region close to the neutral stability curve (see figure $7 d$ ). This rapid decrease of $k_{z}$ corresponds to the boundary separating two- and three-dimensional secondary instabilities, which agrees well with the results of Liu et al. who reported two-dimensional flows mainly close to the threshold of the primary instability (see figure $7 a$ ). In this small region, the $\gamma_{1}$ waves undergo a subharmonic two-dimensional instability $\left(\varphi=\frac{1}{2}\right)$. At low frequency and large Reynolds number, the instability is also found to be two-dimensional $\left(k_{z}=0\right)$ but corresponds to an incommensurate mode $(\varphi \in] 0, \frac{1}{2}[)$. This provides an indication that the boundary 

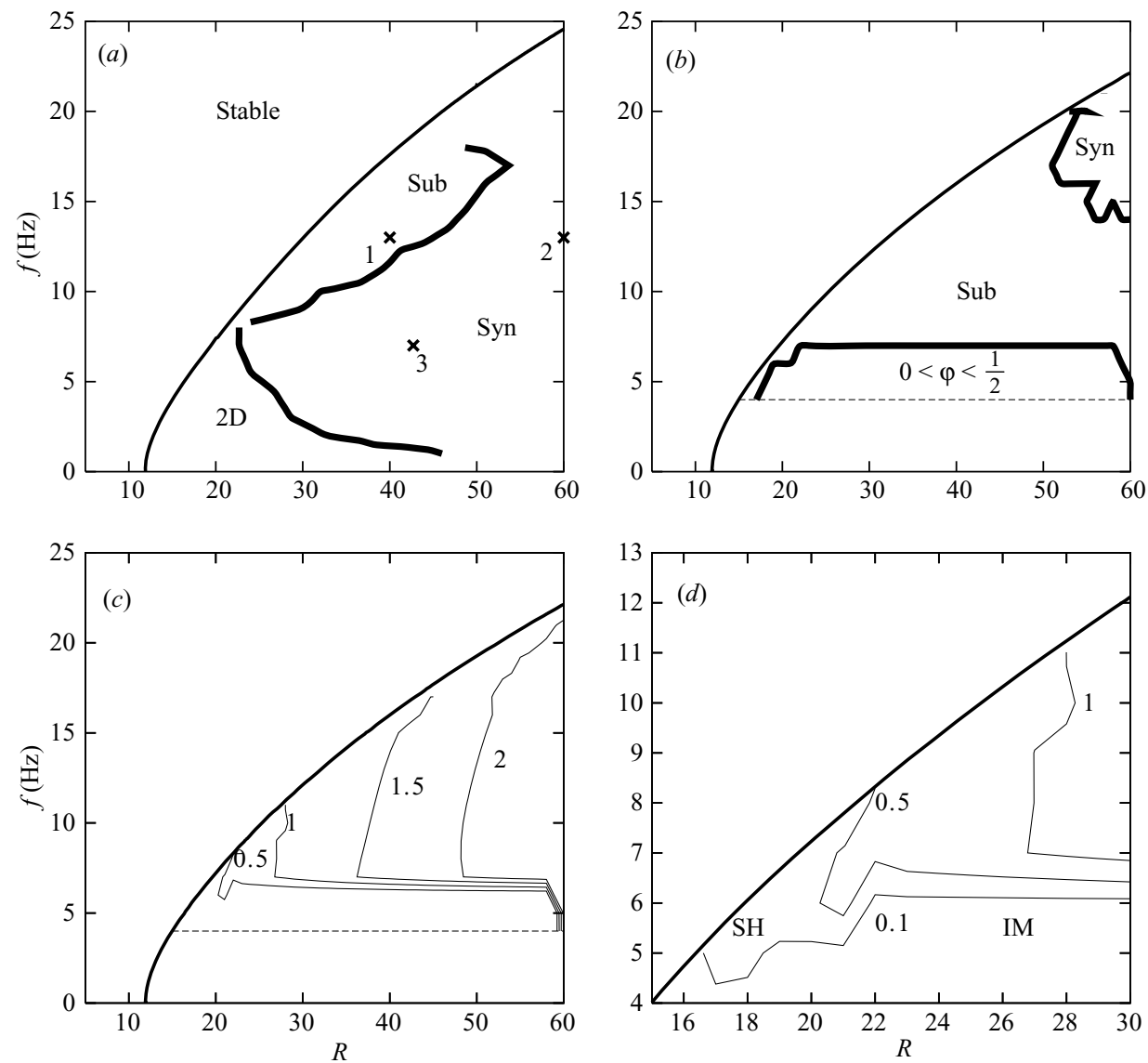

FIGURE 7. Stability of the $\gamma_{1}$ waves to three-dimensional modes as a function of the Reynolds number $R$ and the frequency $f$ for $\beta=4^{\circ}$ and $\Gamma=2340$ (Liu et al. 1995, figure 6). (a) Experimental stability chart. Stability zones are bounded by thick lines: 'two-dimensional' where no three-dimensional instability was observed, 'Sub' for three-dimensional subharmonic instability and 'Syn' for three-dimensional synchronous instability. The neutral stability curve is represented by a thin solid line (Orr-Sommerfeld analysis). Crosses refer to parameter sets reported in table 1. (b) Detuning parameter, where the synchronous (Syn) and subharmonic (Sub) instability regions correspond to $\varphi=0$ and 0.5 , respectively. (c) Wavenumber $k_{z}$ of the fastest growing transverse modulation (in $\mathrm{cm}^{-1}$ ). $(d)$ Enlargement of $(c)$ : 'SH' subharmonic two-dimensional instability $\left(\varphi=\frac{1}{2}\right)$, 'IM' incommensurate modulated two-dimensional mode $\left(0<\varphi<\frac{1}{2}\right)$. Dashed lines in $(b, c)$ indicate the limit $(4 \mathrm{~Hz})$ of the computations. Results presented in $(b-d)$ have been obtained using the regularized model.

between two- and three-dimensional flows may exist and is not an experimental artifact due to finite-size effects. At low frequency and large Reynolds number, Floquet stability analysis of $\gamma_{1}$ waves predicts a two-dimensional region wider than reported in experiments, which can be understood if one keeps in mind that $\gamma_{2}$ waves are likely to develop in that region of the parameter plane in place of $\gamma_{1}$ waves, the stability of which is considered in this section.

As already mentioned, computations predict an overwhelming presence of the subharmonic scenario $\left(\varphi=\frac{1}{2}\right)$ whereas Liu et al. observed it only close to the neutral stability curve at large frequencies and large Reynolds numbers. In fact, our computations predict a region of synchronous three-dimensional instability at 


$\begin{array}{cccccccc}\text { Set } & R & \beta \text { (deg.) } & \Gamma & f(\mathrm{~Hz}) & k & c & \langle h\rangle \\ 1 & 40.0 & 4.0 & 2340 & 13 & 1.565 & 0.824 & 0.987 \\ 2 & 60.0 & 4.0 & 2340 & 13 & 1.494 & 0.689 & 0.970 \\ 3 & 42.7 & 4.0 & 2340 & 7 & 0.953 & 0.703 & 0.975 \\ 4 & 48.0 & 6.4 & 2002 & 10 & 0.980 & 0.628 & 0.965\end{array}$

TABLE 1. Dimensionless wavenumber $k$, phase speed $c$ and averaged thickness $\langle h\rangle$ of the computed $\gamma_{1}$ waves corresponding to experimental conditions by Liu et al. (1995). Figures 7 and 11 in that reference correspond to sets 3 and 4, respectively. The constant mean flow rate condition $\langle q\rangle=1 / 3$ was enforced. Parameters are the Reynolds number $R$, the inclination $\beta$, the Kapitza number $\Gamma$ and the forcing frequency $f$.
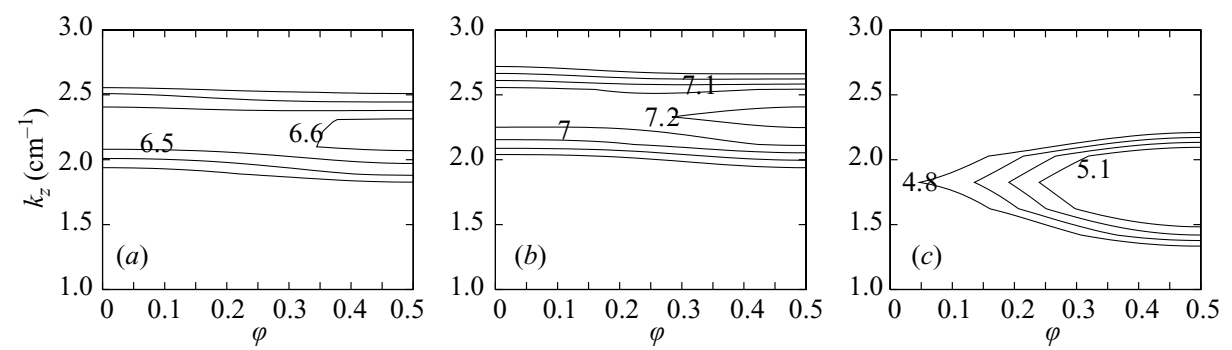

FIGURE 8. Maximum growth rate in $\mathrm{s}^{-1}$ as function of the detuning parameter $\varphi$ and the transverse wavenumber $k_{z}$ in $\mathrm{cm}^{-1}$, computed with the different models for set $2\left(k_{x}=3.2 \mathrm{~cm}^{-1}\right)$ : $(a)$ complete, $(b)$ regularized, $(c)$ simplified.

large Reynolds numbers only when using the regularized model. Figure 8 shows the isocontours of the growth rate $\varsigma_{r}$ of the fastest growing perturbation in the $(\varphi$, $k_{z}$ )-plane for the three models, corresponding to the set 2 of table 1. Similar results (not shown) have been obtained with parameter set 3. Again, the simplified model predicts longer spanwise wavelengths than both the complete and the regularized models. Moreover, figure $8(a, b)$ shows that $\varsigma_{r}$ varies very little with the detuning parameter $\varphi$. Indeed, for the complete and the regularized models, the growth rates for $\varphi=0$ and $\varphi=\frac{1}{2}$ are close to each other so that the instability is not selective. On the other hand, the simplified model is more selective (see figure $8 c$ ) and clearly predicts a subharmonic instability. This result again shows the subtle role of the second-order inertia terms in the pattern selection.

The direct correspondence between results from Floquet analysis and the experiments is based on three assumptions. First, the $\gamma_{1}$ waves emerge from the primary instability. Second, a broadband white noise is assumed. As indicated by Liu et al., the irregularities at the entrance section are time-independent and preferentially trigger in-phase modulations of the evolving three-dimensional patterns. Therefore experimental noise may contain a large amount of in-phase perturbation, which may trigger the synchronous instability instead of the subharmonic mode, given that they have growth rates close to each other. Third, Floquet analysis assumes that two-dimensional waves saturate before the onset of the three-dimensional instability. Precisely because inlet noise may contain significant spanwise perturbations, threedimensional instabilities may arise before the saturation of the two-dimensional waves is achieved. Such a sensitivity to inlet conditions can only be checked by direct numerical simulations of the models. 


\section{Two-dimensional simulations of three-dimensional flows}

In this section we perform time integrations of the complete model, the regularized model (6.2) and the simplified model ((6.2) with $\left.\mathscr{G}_{x}=1\right)$. Periodic boundary conditions in both $x$ - and $z$-directions are imposed. This allows us to take advantage of the good convergence properties of spectral methods. A pseudo-spectral scheme has been developed, with derivatives evaluated in Fourier space and nonlinearities in physical space. The time dependence is accounted for by a fifth-order Runge-Kutta scheme, which allows control of truncation errors by difference with an embedded fourth-order scheme (Press et al. 1992). In practice, the time step is adapted to limit the relative error on each variable to $10^{-4}$. The explicit character of the algorithm makes it easy to implement the different models. The computational domain of size $L_{x} \times L_{z}$ is discretized as a lattice of $M \times N$ regularly spaced grid points with coordinates $x_{i}=i L_{x} / M$ and $z_{j}=j L_{z} / N$. The three-dimensionality of the waves is evaluated through

$$
\begin{aligned}
& E_{x}(t) \equiv \frac{1}{M N} \sum_{j=1}^{N}\left(\sum_{m=1}^{M / 2-1}\left|a_{m}\left(z_{j}, t\right)\right|^{2}\right)^{1 / 2}, \\
& E_{z}(t) \equiv \frac{1}{M N} \sum_{i=1}^{M}\left(\sum_{n=1}^{N / 2-1}\left|b_{n}\left(x_{i}, t\right)\right|^{2}\right)^{1 / 2}
\end{aligned}
$$

where the spatial Fourier coefficients $a_{m}$ and $b_{n}$ are defined by

$$
\begin{aligned}
& a_{m}(z, t)=\sum_{i=0}^{M / 2-1}\left[h\left(x_{2 i}, z, t\right)+\mathrm{i} h\left(x_{2 i+1}, z, t\right)\right] \exp [2 \pi \mathrm{i} m i /(M / 2)], \\
& b_{n}(x, t)=\sum_{j=0}^{N / 2-1}\left[h\left(x, z_{2 j}, t\right)+\mathrm{i} h\left(x, z_{2 j+1}, t\right)\right] \exp [2 \pi \mathrm{i} n j /(N / 2)],
\end{aligned}
$$

and where $\mathrm{i}$ stands for the imaginary unit. $E_{x}$ and $E_{z}$ are the streamwise and the spanwise energy of deformations (Joo \& Davis 1992; Press et al. 1992).

Owing to the spatial periodicity in the streamwise direction, our simulations physically correspond to a closed flow for which the fluid leaving the downstream border of the computational domain is reinjected at the upstream boundary. The mass is therefore conserved in the domain so that the space-averaged film thickness remains constant and is equal to the initial flat-film thickness. In experiments, the fluid accelerates and film thinning is observed so that the time average of the thickness decreases downstream, whereas the time average of the flow rate is conserved and is equal to its value at the inlet, $1 / 3$. Therefore in order to account for the acceleration of the flow observed in experiments, in our simulations the uniform thickness at the initial time is set to the mean thickness $\langle h\rangle<1$ of the two-dimensional travelling waves at the forcing frequency, which are obtained using Auto97 when a constant averaged flow rate $\langle q\rangle=1 / 3$ is enforced, which ensures that the amount of liquid in our computational domain lying under the corresponding travelling waves is appropriate. Since the local flow rate varies as the cube of the local film thickness, this requirement can be crucial in recovering experimental results. The development of two-dimensional waves undergoing three-dimensional instabilities is simulated by enforcing the initial condition

$$
h(x, z, 0)=\langle h\rangle+A_{x} \cos \left(2 \pi n_{x} x / L_{x}\right)+A_{z} \cos \left(2 \pi n_{z} z / L_{z}\right)+A_{\text {noise }} \tilde{r}(x, z),
$$


(a)

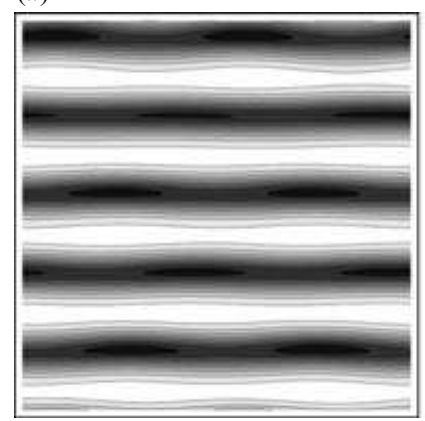

(b)

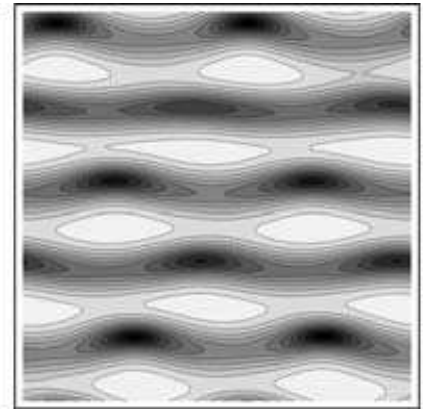

(c)

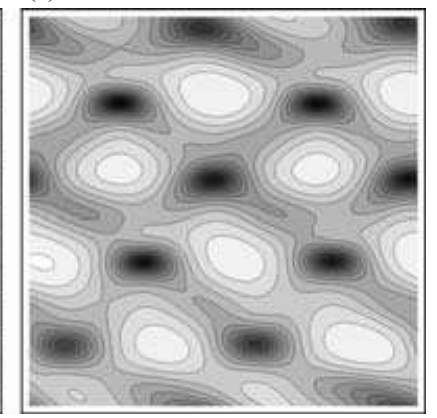

FIGURE 9. Snapshots of free-surface deformations giving rise to a herringbone pattern, computed for the parameter set 1 (see table 1) with the regularized model at different times: (a) $t=150,(b) t=175$, (c) $t=195$. Isothickness contours are separated by an elevation step of 0.06 . The numbers of grid points are $M \times N=128 \times 64$ and $L=2 n_{x} \pi / k$. Amplitudes of the initial periodic forcing are $A_{x}=0.1, A_{z}=0$ and $A_{\text {noise }}=10^{-3}$, with $n_{x}=5$. Dark and bright zones stand for depressions and elevations, respectively.

where $A_{x}, A_{z}, A_{\text {noise }}$ are small amplitudes, $n_{x}, n_{z} \in \mathbb{N}$ represent the number of sinusoidal waves in each direction, and $\tilde{r}$ is a random function with values in the interval $[-1,1]$. The last term of $(8.2)$ accounts for ambient white noise. In the following we take $A_{\text {noise }}=10^{-3}$. In most cases, the aspect ratio of the computational domain is set to unity and $L_{x}=L_{z} \equiv L$. The value of $L$ must be taken large enough to allow complex flow dynamics. The general form of (8.2) enables us to explore a wide range of experimental results on three-dimensional waves emerging from twodimensional waves. In the following, we consider three-dimensional modulations of $\gamma_{1}$ waves, $\gamma_{2}$ waves, and natural waves.

\subsection{Three-dimensional modulations of $\gamma_{1}$ waves}

We first consider the transition from two-dimensional $\gamma_{1}$ waves to three-dimensional patterns, which corresponds to the experimental results by Liu et al. (1995). Their well-controlled experiments will also serve as a benchmark for a systematic evaluation of the accuracy and usefulness of the different models.

Liu et al. have imposed a spanwise-uniform time-periodic forcing at the inlet. In order to mimic their experiments, we choose initial conditions corresponding to sinusoidal two-dimensional waves plus small white noise $\left(A_{z}=0\right.$ and $\left.A_{\text {noise }} \ll A_{x}\right)$. $L$ is set equal to five times the wavelength $2 \pi / k$ of the precursor two-dimensional travelling wave, i.e. $n_{x}=5$. The values of the parameters for the different numerical experiments are indicated in table 1 . We start by considering flow conditions for an inclination angle $\beta=4^{\circ}$ and Kapitza number $\Gamma=2340$ (sets $1-3$ in table 1 and in figure $7 a$ ). Each chosen couple (frequency, Reynolds number) is indicated by a cross in figure $7(a)$. Set 1 corresponds to the region of the plane $(f, R)$ where herringbone patterns were observed experimentally, i.e. subharmonic instability. Simulations of the complete, regularized and simplified models agree with both the Floquet analysis and the experimental data by showing the presence of staggered crests and troughs. Isothickness contours of the wave patterns are shown at different times in figure 9 for the regularized model: at the final stage (figure $9 c$ ), the film evolves towards a staggered arrangement of smooth and large bumps, and thin and deep depressions, which agrees qualitatively with the experimental observations. 
(a)

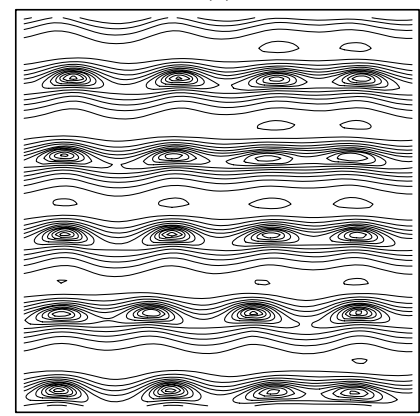

(b)

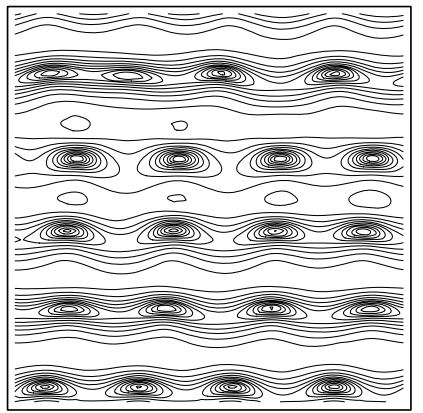

(c)

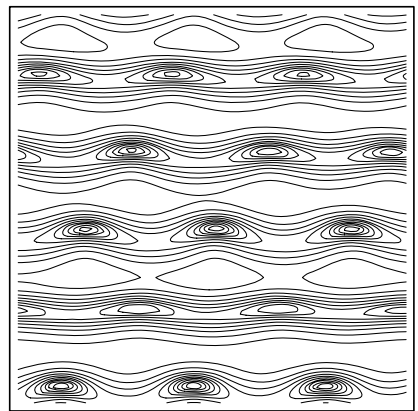

FiguRE 10. Snapshots of free-surface deformations computed for parameter set 2 at $E_{z} \approx 0.05$ for the three models: $(a)$ complete, $t=125 ;(b)$ regularized, $t=125 ;(c)$ simplified, $t=155$. Isothickness contours are separated by a level difference of 0.08 . See also the caption of figure 9. The size of the computational domain is $9.8 \times 9.8 \mathrm{~cm}$. Note that the shading have been removed for clarity.

Using parameter set 2, we move to the region in figure $7(a)$ where synchronous secondary instability has been reported by Liu et al. (1995) whereas the Floquet analysis predicts a subharmonic instability (compare figure $7 a$ to figure $7 b$ ). Time integrations of the different models, given in figure 10 for the same spanwise deformation energy $E_{z}$, disagree: the complete model (panel $a$ ) shows a sideband instability, $\varphi \ll 1$, leading to a synchronous pattern while the simplified model (panel $c$ ) gives staggered troughs and more deformed crests indicating a subharmonic instability, $\varphi=\frac{1}{2}$. Solution to the regularized model (figure 10b) corresponds to a combination of synchronous and staggered modulations, while appearing closer to the complete model's solution (and experimental observations) than to that of the simplified model: spanwise and streamwise wavelengths have values close to each other (four spanwise modulations for the complete and regularized model, in contrast with three for the simplified one). This is in line with the fact that, as seen in figure $8(a, b)$, the secondary instability is not selective for parameter set 2 . On the other hand, as expected from the linear prediction (figure $8 c$ ), the simplified model clearly selects the subharmonic instability, ending in a staggered pattern (figure 10c). Similar behaviours of the three models (not shown here) have been also found for parameter set 3 .

Parameter set 4 of table 1 corresponds to a more pronounced inclination angle $\left(\beta=6.4^{\circ}\right)$ and thus to a smaller Kapitza number $(\Gamma=2002)$. Our simulations indicate that, if the initial excitation is spanwise uniform $\left(A_{z}=A_{\text {noise }}=0\right)$, the twodimensional steady state corresponds to an oscillatory mode instead of a travelling wave. This is illustrated in figure 11 by plotting in $(a)$ the time evolution of the streamwise deformation energy $E_{x}$ and in $(b)$ the wave profiles at two different times corresponding to a maximum (label ' 1 ') and a minimum (label ' 2 ') of $E_{x}$ during one oscillating period. Such an oscillatory mode has been numerically observed by Ramaswamy et al. (1996) who called this regime quasi-periodic. The direct numerical simulations of the Navier-Stokes equations indicate that the quasi-periodic regime is widely present in the case of a vertical plane when the Reynolds number becomes large. This behaviour is generated by the destabilization of the existing limit cycle and can be predicted by looking at the maximum growth rate of Floquet perturbations, the imaginary part of which was also found to be positive.

The wave patterns for the different models are shown in figure 12 . We see that both the complete and the simplified models yield staggered patterns whereas the 

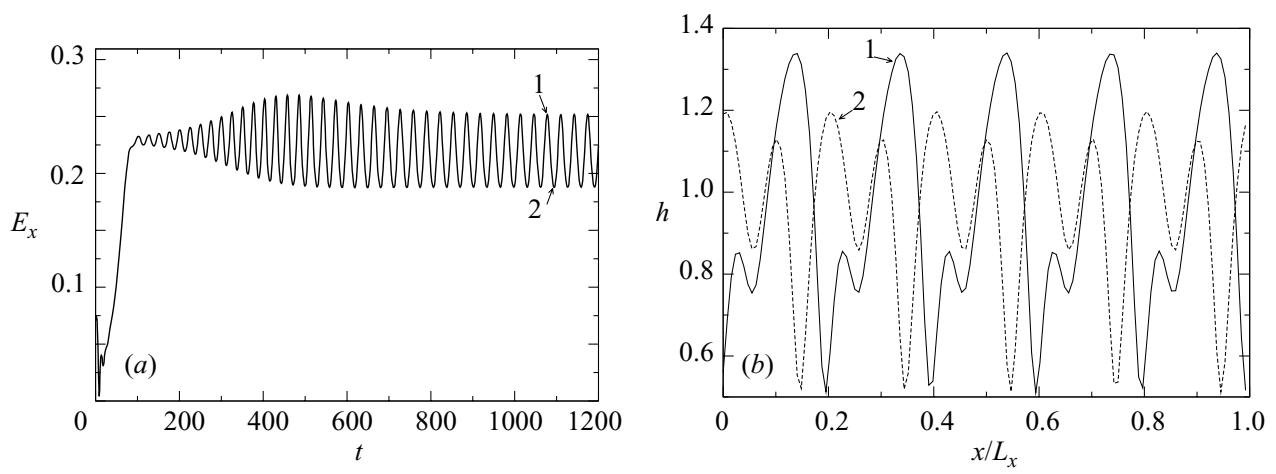

FiguRE 11. (a) Energy of streamwise deformations $E_{x}$ computed for parameter set 4 as a function of time; $(b)$ corresponding two-dimensional wave profiles. The complete model has been used for computations and $A_{x}=0.1, A_{z}=0, A_{\text {noise }}=0, n_{x}=5, L=2 n_{x} \pi / k$ for the initial conditions.

(a)

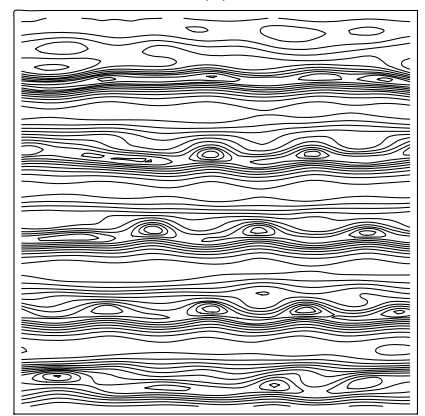

(b)

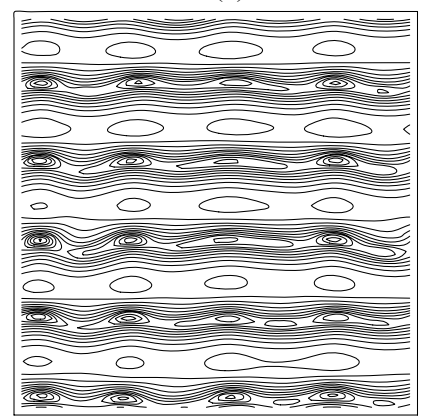

(c)

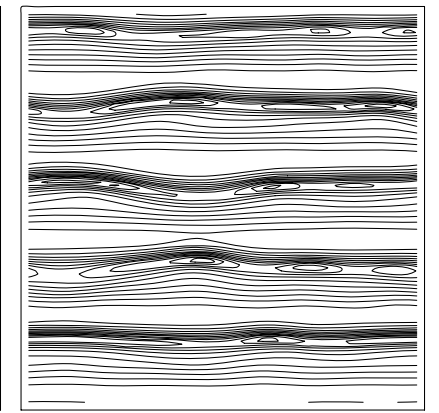

FIGURE 12. Free-surface deformations computed for parameter set 4 at $E_{z} \approx 0.05$ for the three models: $(a)$ complete, $t=345 ;(b)$ regularized, $t=305 ;(c)$ simplified, $t=295$. Isothickness contours are separated by an elevation step of 0.06 . Amplitudes of the initial forcing are $A_{x}=0.2, A_{z}=0$ and $A_{\text {noise }}=10^{-3}$.

regularized model yields a synchronous pattern, in agreement with experimental observations. In fact, it appears that the onset of the three-dimensional pattern is strongly influenced by the presence of the two-dimensional oscillatory mode and then by the exchange of energy between this mode and the three-dimensional instability mode. This exchange depends on the initial conditions and in particular on the amplitude $A_{x}$ of the initial streamwise modulations. Figure 13 shows three-dimensional wave patterns computed with the regularized model for two different values of $A_{x}$. Significant qualitative differences can be noted when comparing them to figure $12(b)$ : at low initial amplitude $A_{x}=0.1$, the final transverse modulations seem to have longer wavelengths than at the larger values of $A_{x}=0.2$ and $A_{x}=0.3$. In addition, crests display out-of-phase modulations whereas modulations are more in-phase when the initial amplitude $A_{x}$ is increased. Time evolution of the energies $E_{x}$ and $E_{z}$ is displayed in figure 14 . When $A_{x}=0.1$, the system approaches the unstable stationary wave solution and remains close to it for a long time. Therefore, the Floquet analysis still applies and the staggered pattern obtained corresponds to the predicted subharmonic instability. This is no longer the case for larger values of $A_{x}$ where the modulation of the two-dimensional wave train occurs prior to the 
(a)

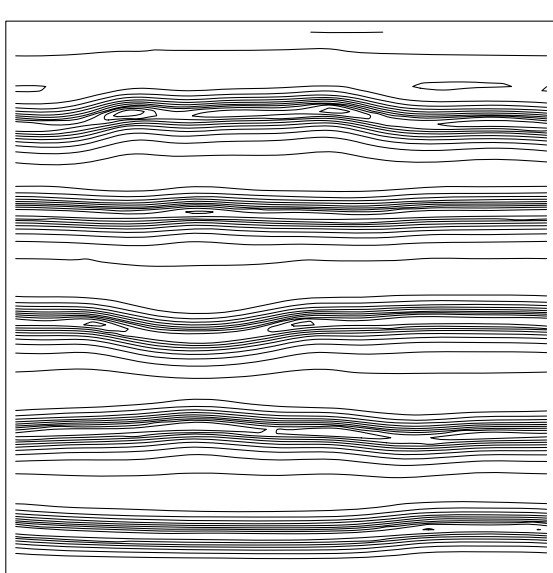

(b)

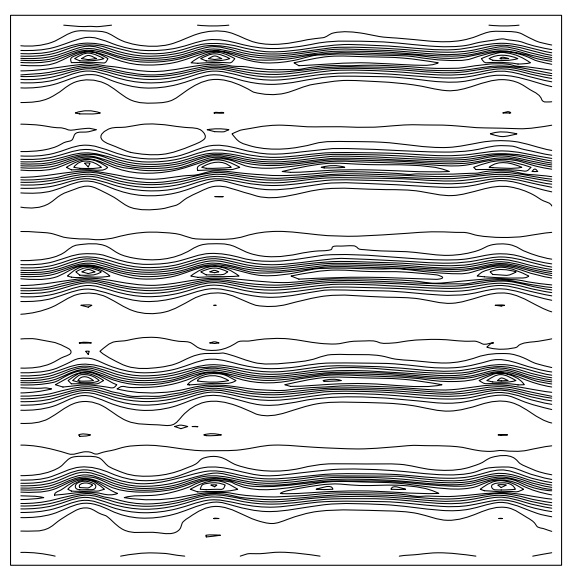

FIGURE 13. Free-surface deformations computed for parameter set 4 (regularized model) at $E_{z} \approx 0.05\left(A_{z}=0\right.$ and $\left.A_{\text {noise }}=10^{-3}\right):(a) A_{x}=0.1$ and $t=300,(b) A_{x}=0.3$ and $t=220$.
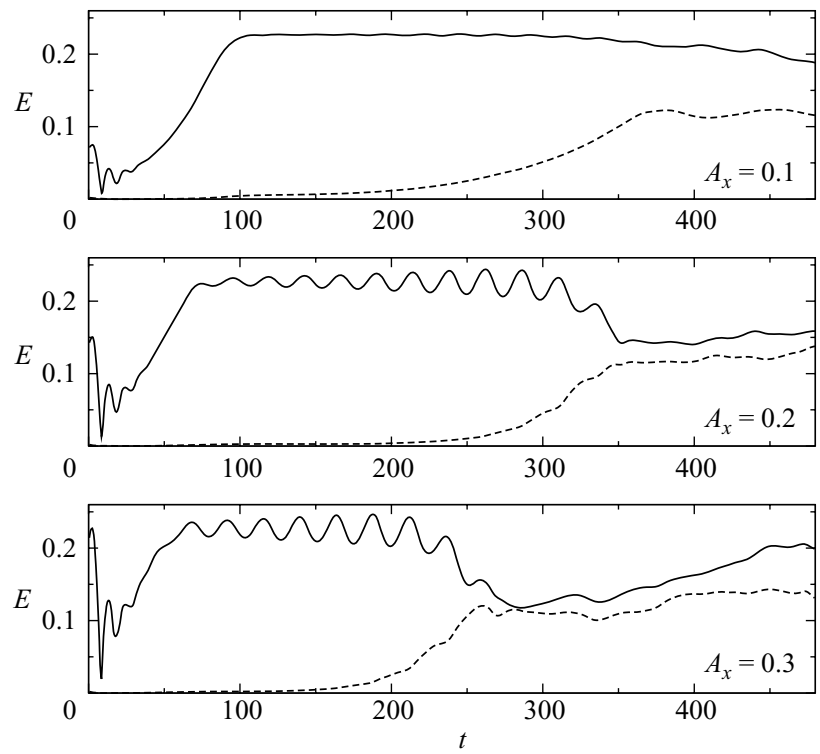

FiguRE 14. Deformation energies computed for parameter set 4 using the regularized model (6.2) and various values of $A_{x}$. Solid and dashed lines correspond to $E_{x}$ and $E_{z}$, respectively. Figures $13(a), 12(b)$ and $13(b)$ correspond to pictures taken at times when $E_{z}$ crosses the level 0.05 .

development of the three-dimensional instability. The observed synchronous pattern is thus the complex result of two ingredients: the growth of two-dimensional oscillations and the three-dimensional instability.

We have already noticed how pattern formation is sensitive to the initial conditions, due to the poor selectivity of the secondary instability. In order to mimic the effect of possible inlet inhomogeneities in our simulations, we have added an $x$-independent noise $\tilde{r}^{\prime}(z)$ to the initial condition (8.2), whose amplitude $A_{\text {noise }}^{\prime}$ represents the inlet roughness. A realistic estimate of about $1 \mu \mathrm{m}$ roughness gives an amplitude of $A_{\text {noise }}^{\prime}=0.01$ for a typical film thickness of $100 \mu \mathrm{m}$. Figures 15 and 16 display results 
(a)

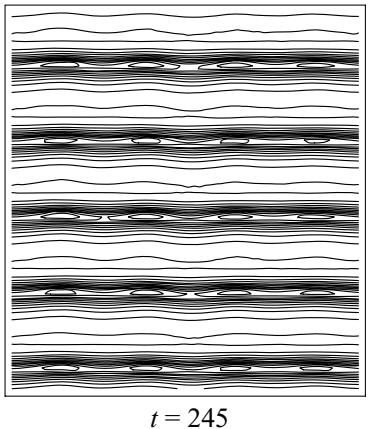

(b)

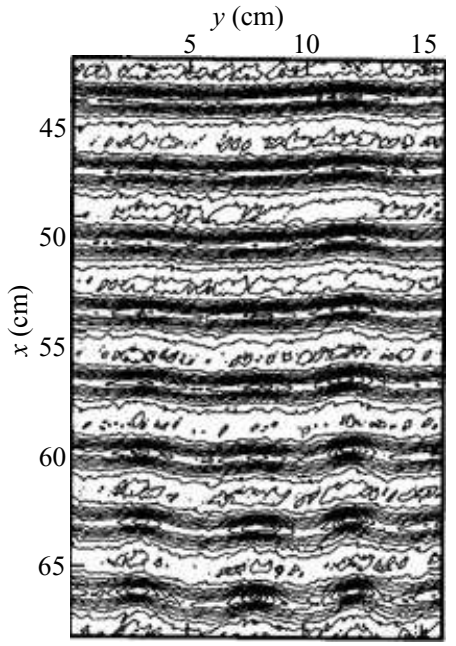

(c)

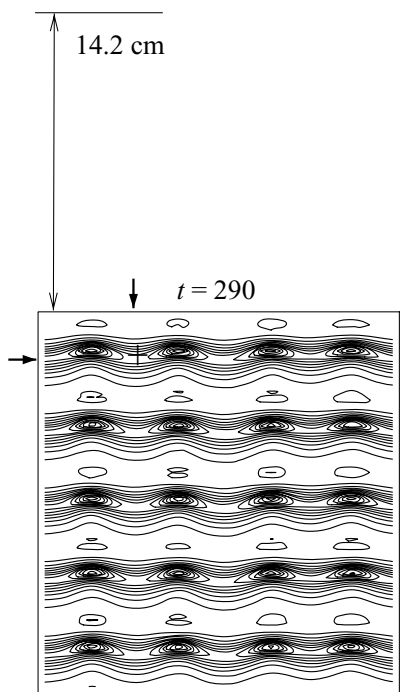

FIGURE 15. $(a, c)$ Snapshots of the film free surface obtained using the regularized model $(6.2)$ at two different times, along with $(b)$ the experimental picture reused with permission from Jun Liu, Physics of Fluids, vol. 7, p. 55 (1995). Copyright 1995, American Institute of Physics. Parameters correspond to set 3 in table $1 . A_{x}=0.2, n_{x}=5, A_{z}=0, L=2 n_{x} \pi / k, A_{\text {noise }}=10^{-3}$ : an $x$-independent noise with amplitude $A_{\text {noise }}^{\prime}=10^{-2}$ is added to mimic the effect of wall roughness. The size of the computational domain is $148 \times 148 \mathrm{~mm}$. Isothickness contours are separated by an elevation step of 0.06 . The location of a saddle point in $(c)$ (see text) is indicated by a cross and two arrows.

(a)

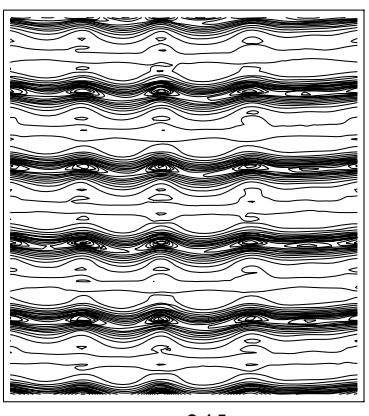

$t=245$ (b)

$y(\mathrm{~cm})$

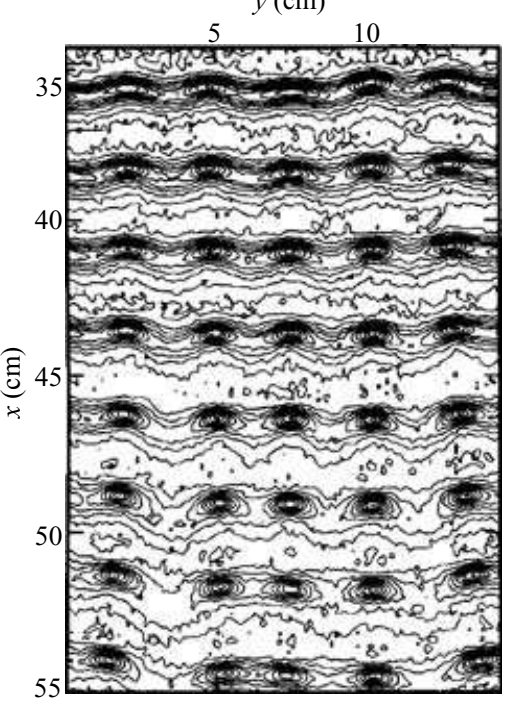

(c)

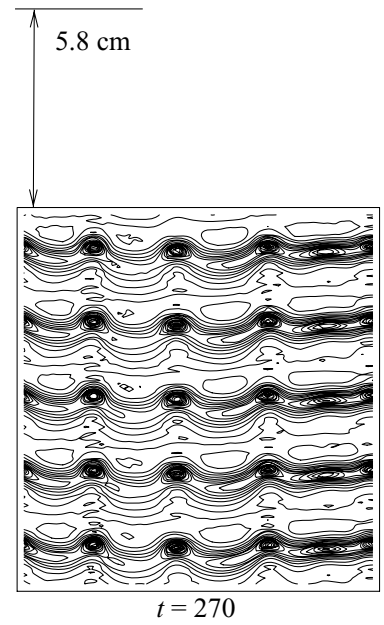

FIGURE 16. As figure 15 but with parameter set 4. The experimental picture in panel (b) is reused with permission from Jun Liu, Physics of Fluids, vol. 7, p. 55 (1995). Copyright 1995, American Institute of Physics. The size of the computational domain is $118 \times 118 \mathrm{~mm}$. Isothickness contours are separated by an elevation step of 0.08 . 
obtained with the regularized model, compared to those obtained experimentally (Liu et al. 1995, figures 7 and 11). They show the influence of such a perturbation, which effectively biases the evolution in favour of the synchronous instability. To facilitate comparisons with the experimental results, numerical snapshots are separated in the vertical direction by the distance covered by the waves between the two times at which the snapshots have been taken (roughly $14.2 \mathrm{~cm}$ and $5.8 \mathrm{~cm}$ in the case of figures 15 and 16 respectively). The agreement with experiments is now reasonable even though, mostly because of the choice of periodic boundary conditions, some differences can still be noticed. The spanwise wavelength selected in the simulation shown in figure 15 seems to be a little smaller than in the experiment ( $37 \mathrm{~mm}$ in comparison to roughly $46 \mathrm{~mm}$ ), whereas in the case of figure 16 , the simulation and the experiment give essentially the same answer ( $28 \mathrm{~mm}$ compared to $26 \mathrm{~mm}$ ). However, experiments and simulations share common qualitative features. Isothickness contours agree well with each other, and strong modulations of the troughs are observed, whereas the crests remain nearly undeformed, which leads to the formation of isolated depressions. In particular, as experimentally observed by Liu et al., our numerical simulations indicate the formation of local saddle points on the wave pattern corresponding to minima in the spanwise direction and maxima in the streamwise direction (see figure $15(c)$ where one such saddle point is indicated by a cross). Liu et al. have measured the difference in height between the minima of the thickness at a trough and the height of the nearby saddle point. They called it 'trough transverse modulation amplitude', denoted $\Delta h_{\min }(x)$. From the measurement of $\Delta h_{\min }(x)$ at different locations for the experimental data corresponding to parameter set 3, i.e. their figure 7 and our figure 15, they computed a spatial growth rate of approximately $0.11 \mathrm{~cm}^{-1}$. Following a similar procedure, we define $\Delta h_{\min }(t)$ as the height difference between the minimum of the thickness in the entire computational domain and the closest saddle point at a given time $t$. From the measurement of $\Delta h_{\min }(t)$ in our simulation, we found a temporal growth rate of approximately $2.6 \mathrm{~s}^{-1}$, which is converted into a spatial growth rate, $0.125 \mathrm{~cm}^{-1}$, hence of the correct order of magnitude, with the help of the speed of the corresponding two-dimensional $\gamma_{1}$ waves, $20.8 \mathrm{~cm} \mathrm{~s}^{-1}$.

Despite differences between our numerical simulations and experimental conditions, both the synchronous instability and the herringbone patterns observed by Liu et al. (1995) were qualitatively recovered with the complete and the regularized models, whereas the synchronous instability cannot be obtained using the simplified one. This indicates the necessity of taking into account the second-order inertia corrections to reproduce satisfactorily the experimental findings. The regularized model (6.2) therefore seems to be a good compromise between accuracy and simplicity and will be the only one used from now on to compare numerical simulations with experimental findings.

\subsection{Three-dimensional modulations of $\gamma_{2}$ waves}

In this section, we investigate the experimental conditions of Park \& Nosoko (2003) who observed three-dimensional wave patterns emerging from two-dimensional waves of $\gamma_{2}$-type for films of water on a vertical wall. Parameter sets corresponding to the different numerical experiments are given in table 2. Controlling inlet perturbations, Park \& Nosoko (2003) have imposed a spanwise-uniform forcing at a given frequency $f$ and periodic modulations in the spanwise direction by means of regularly spaced needles with period $\lambda_{z, \text { ndl }}$. At $R$ below approximately 40, regular spanwise forcing 


\begin{tabular}{cccccccccc}
\hline Set & $R$ & $\beta($ deg. $)$ & $\Gamma$ & $f(\mathrm{~Hz})$ & $\lambda_{z, \text { ndl }}(\mathrm{mm})$ & $k$ & $c$ & $\langle h\rangle$ & $k_{z}$ \\
5 & 20.7 & 90 & 3375 & 15.0 & 10 & 0.3461 & 0.900 & 0.899 & 0.699 \\
6 & 40.8 & 90 & 3375 & 19.1 & 20 & 0.3845 & 0.714 & 0.912 & 0.377 \\
7 & 59.3 & 90 & 3375 & 17 & 20 & 0.3126 & 0.630 & 0.955 & 0.393
\end{tabular}

TABLE 2. Parameters of the simulations corresponding to experiments on a vertical plane and with pure water at $25^{\circ} \mathrm{C}$ (Park \& Nosoko 2003, figure 7). $\lambda_{z}$,ndl is the spanwise intervals of the needle array and $k_{z}$ is the corresponding dimensionless wavenumber. The dimensionless wavenumber $k$, phase speed $c$ and averaged thickness $\langle h\rangle$ of the corresponding two-dimensional $\gamma_{2}$ waves are also given.

(a)

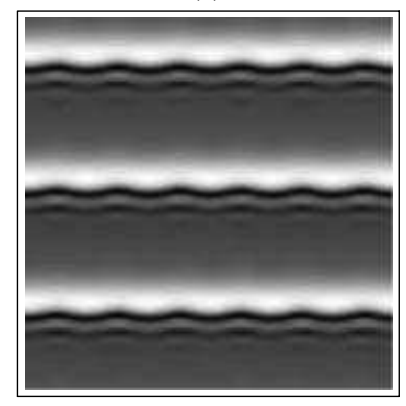

$(b)$

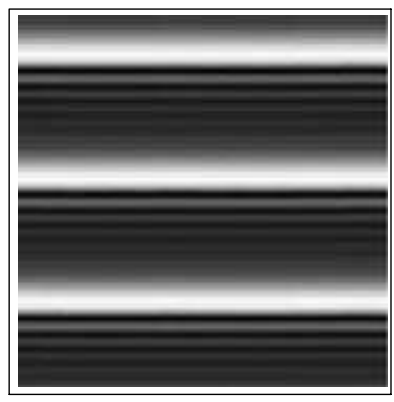

(c)

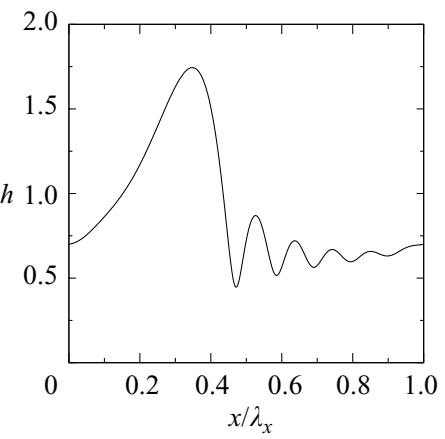

Figure 17. $(a, b)$ Snapshots of the film free surface at times $t=27$ and $t=172$ computed with the regularized model and for set 5 in table $2(R=20.7) . n_{x}=3, n_{z}=6$ and $L=2 n_{x} \pi / k$. The computational domain is $60 \times 60 \mathrm{~mm}$ with $128 \times 128$ grid points. Bright (resp. dark) zones correspond to elevations (resp. depressions). (c) two-dimensional wave profile of $(b)$.

of the waves led to low-level spanwise modulations whereas at $R$ above 40, the waves broke into horseshoe-like solitary waves having curved fronts and long oblique legs. The existence of stationary horseshoe-like waves has been demonstrated experimentally by Alekseenko et al. (2005). The initial conditions (8.2) corresponding to the inlet conditions imposed by Park \& Nosoko and adapted to our simulations are taken as: $A_{x}=0.2, A_{z}=0.05$ and $A_{\text {noise }}=0$.

Figure 17 shows snapshots for parameter set 5 with $R=20.7$. Initial spanwise modulations of length $\lambda_{z, \text { ndl }}=10 \mathrm{~mm}\left(n_{z}=6\right)$ are quickly damped, i.e. $E_{z} \rightarrow 0$, and the pattern evolves to two-dimensional travelling waves, i.e. $E_{x} \rightarrow$ const, the profile of which is given in figure $17(c)$. This corresponds to a $\gamma_{2}$ wave with a large hump preceded by capillary ripples, in accordance with the fact that when the forcing frequency is small, the $\gamma_{1}$ slow waves are not observed. The linear inception region is thus immediately followed by the formation of fast $\gamma_{2}$ waves, that are stable for a while. This is in agreement with the experimental observations, where the inlet forcing is quickly damped. Park \& Nosoko then observed the downstream growth of another mode leading to spanwise-modulated waves with a wavelength roughly equal to $3 \mathrm{~cm}$. Similar modulated $\gamma_{2}$ waves (not shown here) are recovered by increasing the length of the initial spanwise modulations $\lambda_{z, \text { ndl }}$ to $30 \mathrm{~mm}\left(n_{z}=2\right)$. They also decay (with $E_{z} \rightarrow 0$ ) but at a much smaller rate indicating that the wavelength $\lambda_{z}=3 \mathrm{~cm}$ is close to (but still below) the cut-off wavelength for spanwise instability with our regularized model. 
(a)

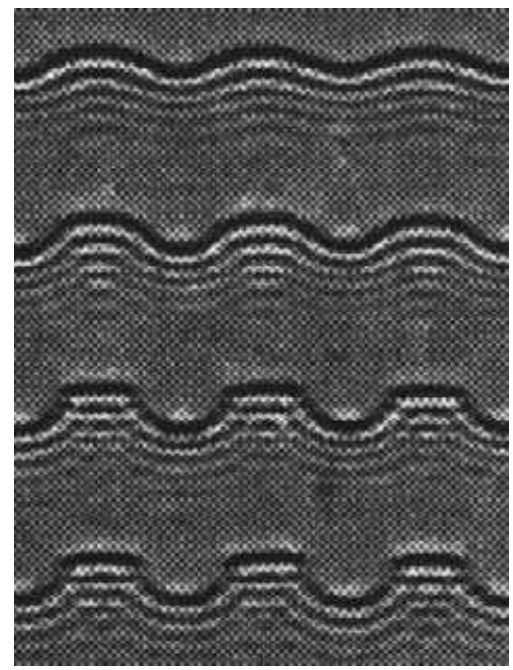

(b)

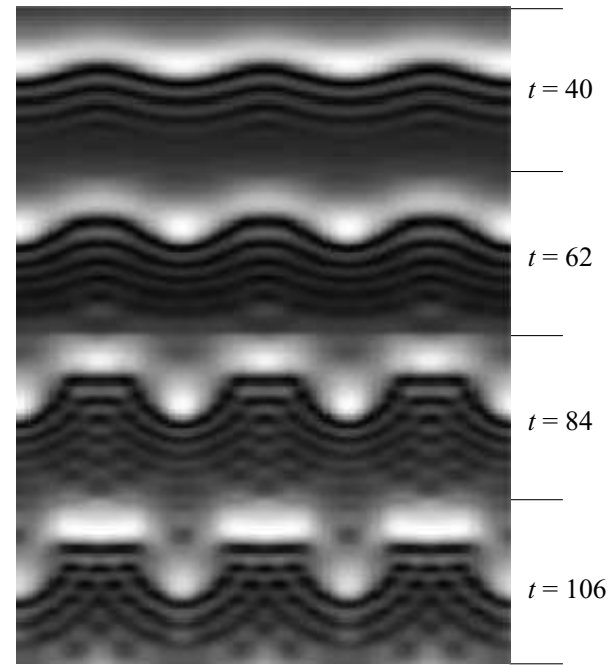

FigURE 18. (a) Experimental picture (real size $60 \times 80 \mathrm{~mm})$ for set $6(R=40.8)$ (Park $\&$ Nosoko 2003 , figure $7 c$, with permission from John Wiley \& Sons, Inc.) (b) Simulations with $n_{x}=3$, $n_{z}=3$ and $L=2 n_{x} \pi / k$. The domain size is $60 \times 60 \mathrm{~mm}$ with $256 \times 256$ grid points. Each of the six wave fronts has been obtained at a different dimensionless time, in an interval of 22 .

Simulation results for a larger Reynolds number $R=40.8$ are presented in figure 18 (parameter set 6) and compared to experimental findings (Park \& Nosoko 2003, figure $7 c$ ). Like for $R=20.7$, we first observe sinusoidal spanwise modulations of the two-dimensional waves. However, they rapidly evolve into rugged modulations, made of nearly flat backs and rounded fronts. To facilitate qualitative comparisons to the spatial evolution observed in experiments, snapshots of only a third of the numerical domain, corresponding to one streamwise wavelength, are displayed in figure 18 at increasing times. The interval of time separating each pair of snapshots roughly corresponds to the travelling of the fronts over a distance equal to one wavelength. Despite our use of periodic boundary conditions, the resemblance with the experimental findings (Park \& Nosoko 2003, figure 7c) is convincing. For instance the chequerboard interference pattern of the capillary waves preceding the flat zones is recovered.

Above $R \approx 40$, Park \& Nosoko (2003) observed a breaking of the modulated fronts leading to horseshoe-like waves. Simulation results for $R=59.3$ are presented in figure 19 (parameter set 7) and compared to the experimental findings (Park \& Nosoko 2003 , figure $7 d$ ). Owing to computational limitations, the computational domain was limited to only one and two wavelengths in the streamwise and spanwise directions respectively $\left(n_{x}=1\right.$ and $\left.n_{z}=2\right)$. Compared to $R=40.8$, the rugged modulations develop faster and do not saturate. Instead, the bulges of the wave front continuously expand into horseshoe shapes, reducing the span of the flat parts at the back. As time proceeds, the legs of the horseshoes extend and split off into dimples, in qualitative agreement with experimental observations. The growth of the spanwise perturbations in our simulation is however faster than in the experiment.

\subsection{Three-dimensional natural waves}

In this section, we study the formation of noise-driven three-dimensional waves in the absence of periodic forcing. To match with the experiments by Alekseenko 
(a)

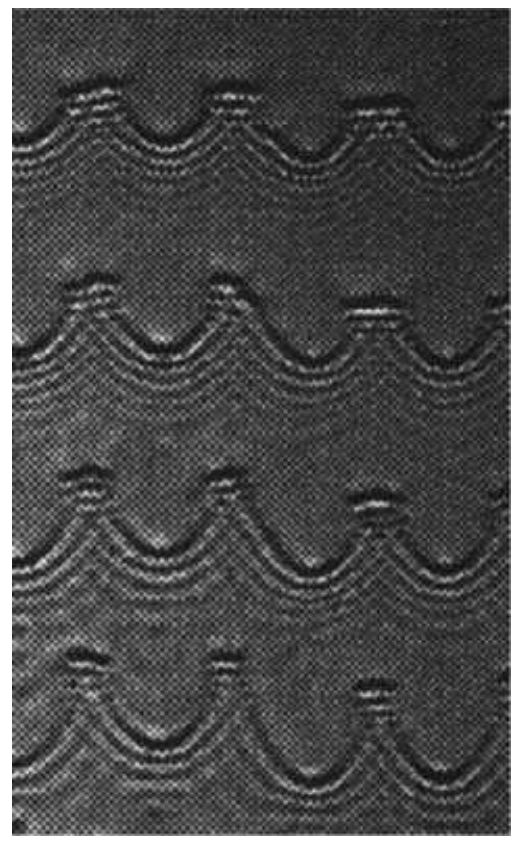

(b)

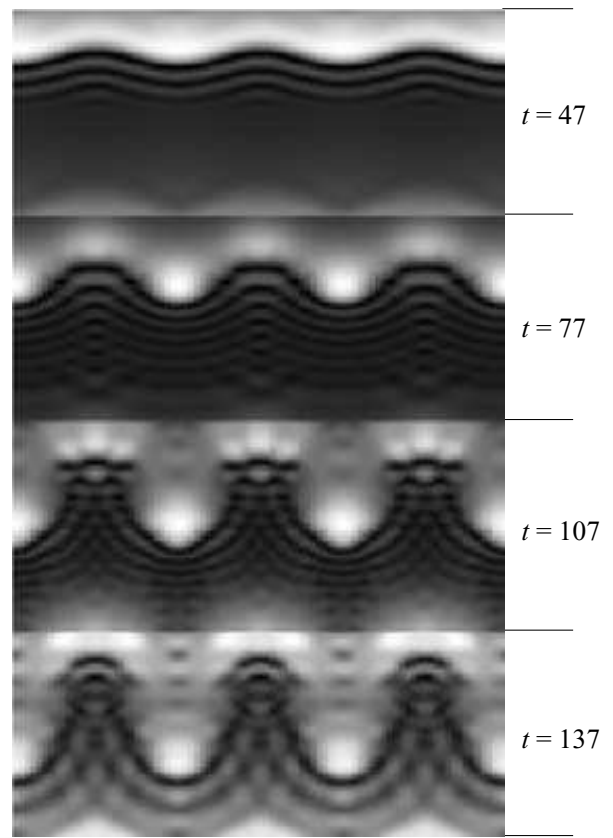

Figure 19. (a) Experimental picture (real size $60 \times 100 \mathrm{~mm})$ for set $7(R=59.3)$ (Park \& Nosoko 2003, figure $7 d$, with permission from John Wiley \& Sons, Inc.) (b) Snapshots of the simulated free surface. The domain size is $40 \times 25 \mathrm{~mm}$ with $256 \times 256$ grid points. Each of the five wave fronts has been obtained at increasing dimensionless times, by interval of 30 .

$\begin{array}{rrcccccc}\text { Set } & R & \beta \text { (deg.) } & \Gamma & \lambda_{x}(\mathrm{~mm}) & k & c & \langle h\rangle \\ 8 & 8 & 75 & 1106 & 40 & 0.15 & 1.322 & 0.906 \\ 9 & 16 & 75 & 1106 & 30 & 0.21 & 1.062 & 0.876 \\ 10 & 45 & 75 & 1106 & 25 & 0.28 & 0.749 & 0.904\end{array}$

TABLE 3. Parameters of the simulations corresponding to experiments on an inclined plane and with a $16 \%$ water-ethanol solution at $25^{\circ} \mathrm{C}\left(\rho=972 \mathrm{~kg} \mathrm{~m}^{-3}, v=1.55 \times 10^{-6} \mathrm{~m}^{2} \mathrm{~s}^{-1}\right.$ and $\sigma=40.8 \times 10^{-3} \mathrm{~N} \mathrm{~m}^{-1}$ ) (Alekseenko et al. 1994, figure 1.6). The two-dimensional wave characteristics $k, c$ and $\langle h\rangle$ have been computed from the wavelength $\lambda_{x}$, which has been estimated by the average streamwise separation of the three-dimensional waves observed in the experimental pictures. See also the caption of table 1 .

et al. (1994), the initial conditions (8.2) need to be chosen with white noise of amplitude $A_{\text {noise }}=10^{-3}$ and $A_{x}=A_{z}=0$. Parameter values for the different numerical experiments are given in table 3 . Snapshots of the free-surface deformation are reported in figure 21 where the three columns correspond to different Reynolds numbers (sets 8-10 of table 3). The experimental pictures obtained by Alekseenko et al. (1994) are shown for reference in figure 20. Each row in figure 21 corresponds to a particular transient regime: first, mostly two-dimensional waves; second, coalescence processes, and finally three-dimensional solitary waves. Both the dimensionless time $t$ and the approximate location of the numerical domain on the experimental plane are given in figure 21. The distance being again estimated from the phase speed $c$ of the two-dimensional waves (see table 3 ). 
(a)

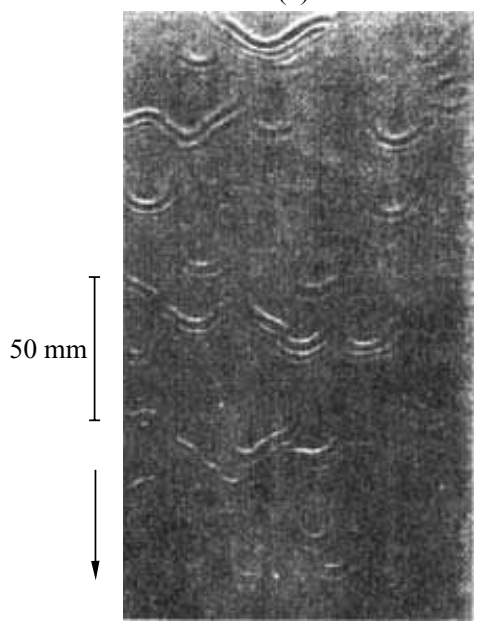

(b)

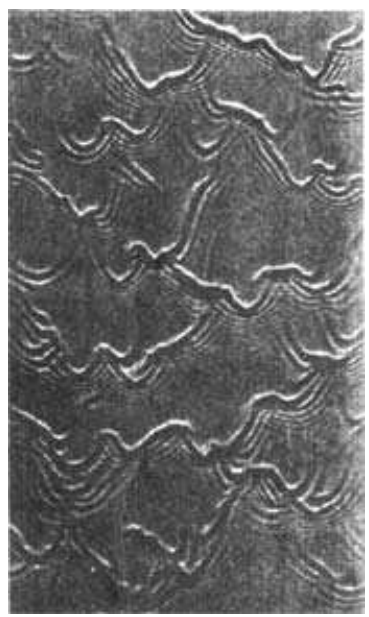

(c)

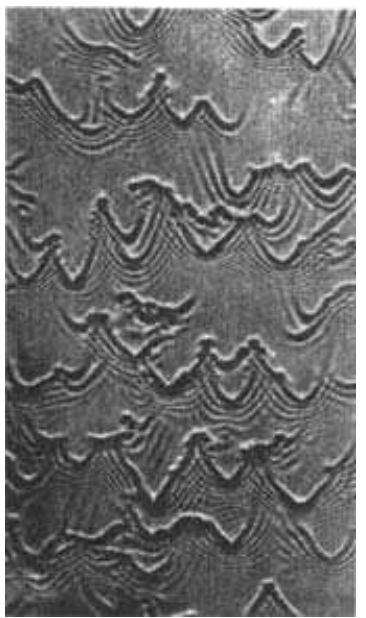

FIGURE 20. Wave patterns obtained experimentally by Alekseenko et al. (1994) (see table 3), courtesy of S. V. Alekseenko.

The large-amplitude waves travel faster, catch up the preceding slower ones and finally absorb them, which explains the coarsening process leading to an increase of the size of the flat zones that separate the waves. The development of capillary ripples in front of the humps is observed and the waves therefore resemble the twodimensional $\gamma_{2}$ waves. Panels $(g, j)$ and $(h, k)$ of figure 21 share features similar to the experimental wave patterns. (For comparison, one should keep in mind that the grey levels represent surface elevation in simulations but surface slope in experiments.) The unsteady experimental pattern is characterized by interacting quasi-steady threedimensional solitary waves separated by portions of constant thickness of length 10 to $50 \mathrm{~cm}$. For $R=8$, the average distance between the solitary waves tends to saturate for $t>890$, which indicates either that solitary waves have reached a fully developed regime, or that the streamwise-periodic conditions are felt. For $R=16$, no fully developed regime has been reached at the end of the simulation, which was run for 1500 time units. In that case, the final stage corresponds to interacting oblique fronts rather than three-dimensional horseshoe-like waves. For $R=45$, the three-dimensional waves tend to form localized structures rather than extended wave fronts as observed for smaller values of $R$. This is in agreement with the results of Alekseenko et al. (1994) and Park \& Nosoko (2003) who observed V-shaped or horseshoe-like solitary waves with a sharp curved front and long backwards tails under similar conditions (see panels $i$ and $l$ ).

\section{Concluding remarks}

In most cases, asymptotic expansions are poorly converging and the Benney expansion is no exception to this rule (Oron \& Gottlieb 2004). If an improvement of the accuracy is achieved by increasing the order of the approximation, this is at the cost of an increased complexity and a reduction of the range of parameters for which comparisons with DNS and experiments are satisfactory. Padé approximant techniques are well known for their ability to extend the radius of convergence of algebraic series. 
SET \# $8(R=8)$

(a) $t=105, l \approx 13 \mathrm{~cm}$

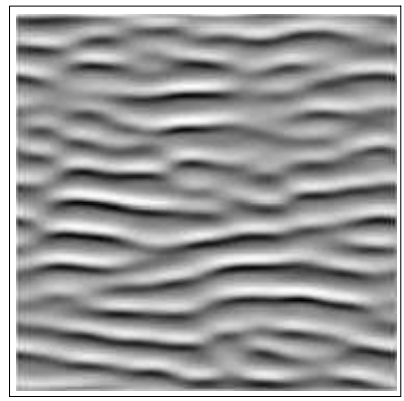

(d) $t=280, l \approx 35 \mathrm{~cm}$

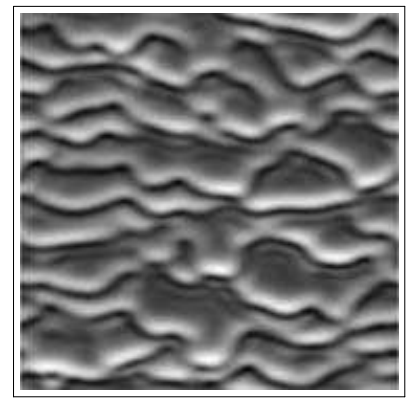

(g) $t=480, l \approx 59 \mathrm{~cm}$

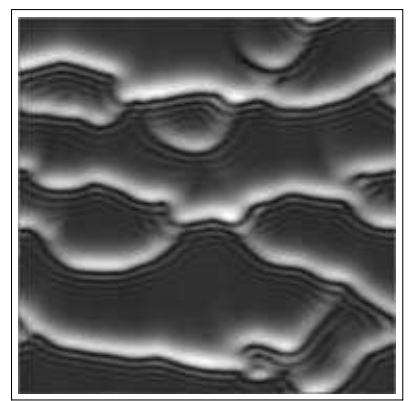

(j) $t=890, l \approx 110 \mathrm{~cm}$

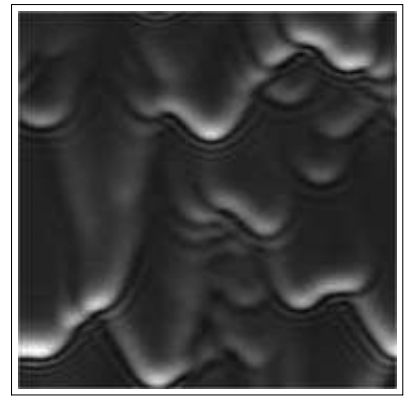

SET \# $9(R=16)$

(b) $t=120, l \approx 13 \mathrm{~cm}$

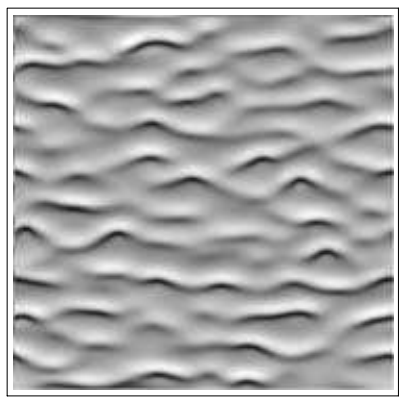

(e) $t=200, l \approx 21 \mathrm{~cm}$

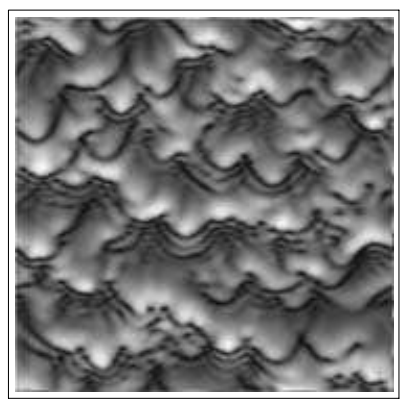

(h) $t=370, l \approx 40 \mathrm{~cm}$

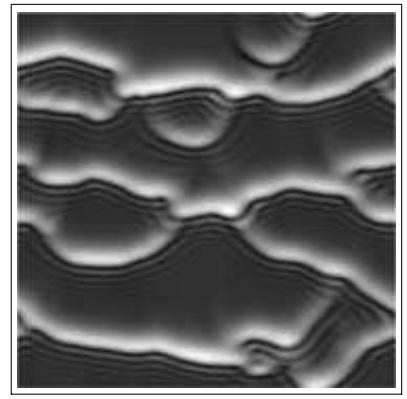

(k) $t=845, l \approx 91 \mathrm{~cm}$

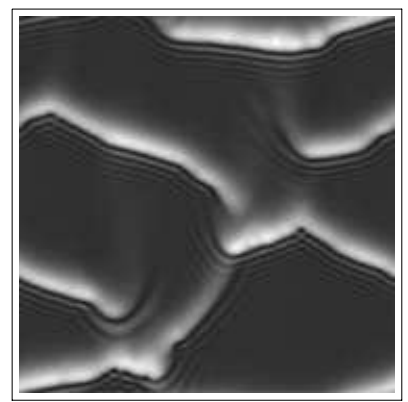

SET \# $10(R=45)$

(c) $t=185, l \approx 16 \mathrm{~cm}$

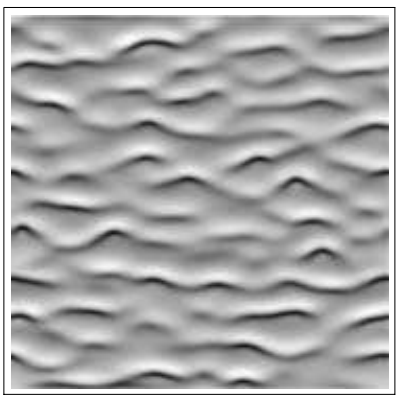

(f) $t=310, l \approx 26 \mathrm{~cm}$

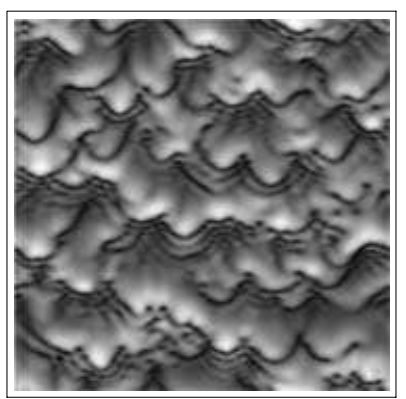

(i) $t=375, l \approx 32 \mathrm{~cm}$

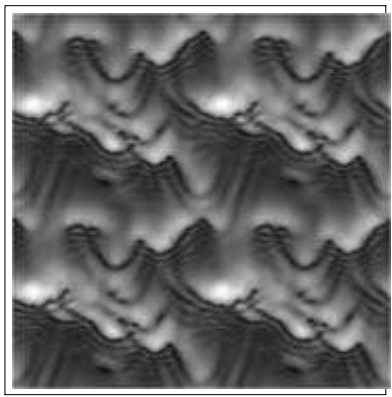

(l) $t=575, l \approx 51 \mathrm{~cm}$

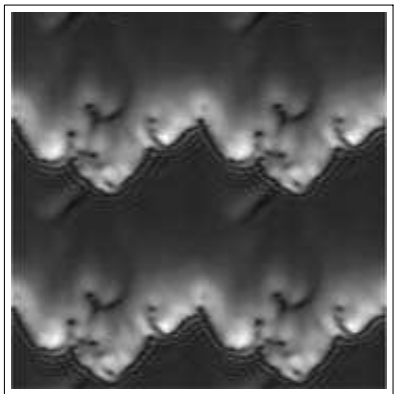

FIGURE 21. Simulations of natural (noise-driven) three-dimensional wave patterns corresponding to the experiments by Alekseenko et al. (1994) (see figure 20). The computational domain is $100 \times 100 \mathrm{~mm}^{2}$ with $256 \times 256$ grid points for set 8 and 9 and $512 \times 256$ for set 10 except for $(i, l)$ where it corresponds to $50 \times 50 \mathrm{~mm}^{2}$ and $256 \times 256$ grid points: the snapshot obtained is repeated four times. $l$ is the estimated distance from the inlet. The bright (dark) zones correspond to elevations (depressions). 
In the case of two-dimensional flows, Ooshida's application of this idea to the Benney expansion remedies the unphysical occurrence of finite-time blow-up of solutions to (3.3) but a quantitative agreement cannot be achieved with experiments for $\delta$ of order unity or larger. We have shown elsewhere that a weighted residual procedure based on a polynomial expansion of the velocity field leads to a twoequation model at order $\epsilon$ in terms of the film thickness $h$ and the flow rate $q$ (Ruyer-Quil \& Manneville 2000). Augmenting the order of the approximation to $\epsilon^{2}$ again leads to a dramatic increase of the complexity yielding a four-equation model in the two-dimensional case and seven equations in the three-dimensional case. A way out can be found by dropping second-order inertial terms while retaining streamwise viscous diffusion effects, thus leading to the simplified model (3.1), (4.1). However, this simplification is done at the cost of a lowering of the order of the approximation. Based on the Padé approximant approach, the first part of this study has been devoted to the derivation of a two-equation model consistent at order $\epsilon^{2}$, aiming at an agreement with DNS and experiments in the largest possible range of parameters.

Focusing on the treatment of inertia terms, our algebraic regularization procedure enabled us to obtain a two-equation model (3.1), (4.15) which is fully consistent with the Benney expansion up to second order. The approach developed here remedies the lack of systemization of the derivations presented in Ruyer-Quil et al. (2005) and Scheid et al. (2005a) where ad-hoc arguments were invoked to treat the case of a film uniformly heated from below. The use of a kind of algebraic preconditioner makes its application much simpler than Ooshida's approach based on differential operators. Our hope is that this Padé-like strategy might be useful for different related problems in lubrication theory for which a careful treatment of inertial effects are of importance, e.g. film flows where mass and heat transfer are involved, films down fibres, and roll waves (Balmforth \& Liu 2004).

Computations of the two-dimensional solitary wave branches of solutions and two-dimensional periodic travelling waves agree quantitatively with laboratory and DNS experiments for the whole range of parameters for which two-dimensional wavy motion is observed. In particular, our models are able to capture the near-wall counterflow observed in the DNS by Malamataris et al. (2002) and in the experiments by Tihon et al. (2003), an effect that might be important when transfer of heat or mass from the substrate are considered.

We have extended our models to include the spanwise dependence in order to study the transition from two-dimensional to three-dimensional flows. A systematic Floquet analysis of the stability of the two-dimensional slow $\gamma_{1}$ waves has been performed, followed by numerical simulations using periodic boundary conditions. Our focus is the description of the three-dimensional wave patterns observed experimentally, with three main objectives: (i) using experimental results as benchmarks for a validation of our models; (ii) reproducing the synchronous and subharmonic transitions from $\gamma_{1}$ waves to three-dimensional patterns found by Liu et al. (1995); (iii) recovering the wave dynamics observed by Park \& Nosoko (2003) in the case of well-controlled spanwise perturbations of fast $\gamma_{2}$ waves, and by Alekseenko et al. (1994) in the case of noise-driven instabilities.

Floquet analysis shows that the secondary three-dimensional instability is not selective, since the maximum growth rate remains nearly unchanged over the whole range $0 \leqslant \varphi \leqslant 1 / 2$ of the detuning parameter. This property makes the threedimensional instability strongly dependent on the initial conditions, and thus prevents one relating unequivocally the results of Floquet analysis to experimental findings. By contrast, numerical simulations have shown good agreement with experimental results by Liu et al. (1995), provided that initial conditions are appropriately tuned. The 
widespread observation of the synchronous instability in experiments could then be attributed to the presence of spanwise non-uniformities at the inlet, favouring in-phase modulations of the wave fronts. In some cases, the three-dimensional patterns emerge from a two-dimensional oscillatory mode rather than from saturated travelling waves, as also observed in direct numerical simulations by Ramaswamy et al. (1996). The competition between the growing two-dimensional modulation and the secondary three-dimensional instability makes the evolution of the film more sensitive to initial conditions. Complex three-dimensional dynamics deep in the nonlinear regime, in particular isolated synchronous depressions (figure 16), rugged-modulated waves (figure 18) as well as horseshoe-like three-dimensional solitary waves (figures 19 and $21 i, l$ ) and oblique solitary waves (figure $21 k$ ) found in our simulations were observed in experiments.

The application of a systematic strategy to the problem of film flows is shown here to lead to systems of equations of reduced dimensionality that capture the physical mechanisms quite faithfully, helping us to highlight the observed dynamics by isolating the important physical effects. Having reliable low-dimensional models at our disposal allows us to attack many questions still open for plain film flows over inclined planes, but also in more difficult cases, for example when heat or mass transfer are involved.

The authors wish to express their gratitude to N. A. Malamataris, M. Vlachogiannis and V. Bontozoglou for providing them with the wave profile corresponding to the full-scale numerical computation of the basic equations and shown in figure 3. C.R.-Q. and B.S. would like to thank Serafim Kalliadasis for stimulating discussions during his stay in Orsay. This study was partly funded by a grant from both French and Belgium research agencies (CNRS/CGRI-FNRS cooperation agreement). B.S. acknowledges funding from the European Commission through the Marie-Curie Training Centre.

\section{Appendix A. Reconstruction of the velocity profile}

Expressions for the polynomials $g_{0}, g_{1}$ and $g_{2}$ used to expand the velocity field are (Ruyer-Quil \& Manneville 2000)

$$
\begin{aligned}
& g_{0}(\bar{y})=\bar{y}-\frac{1}{2} \bar{y}^{2} \\
& g_{1}(\bar{y})=\bar{y}-\frac{17}{6} \bar{y}^{2}+\frac{7}{3} \bar{y}^{3}-\frac{7}{12} \bar{y}^{4}, \\
& g_{2}(\bar{y})=\bar{y}-\frac{13}{2} \bar{y}^{2}+\frac{57}{4} \bar{y}^{3}-\frac{111}{8} \bar{y}^{4}+\frac{99}{16} \bar{y}^{5}-\frac{33}{32} \bar{y}^{6} .
\end{aligned}
$$

Streamlines and velocity profiles displayed in figure 4 were reconstructed from the solutions to the complete and the regularized models using the projection of the streamwise velocity on $g_{0}=\bar{y}-\frac{1}{2} \bar{y}^{2}, g_{1}$ and $g_{2}$ :

$$
u=3 \frac{q-r-s}{h} g_{0}\left(\frac{y}{h}\right)+45 r g_{1}\left(\frac{y}{h}\right)+210 s g_{2}\left(\frac{y}{h}\right) .
$$

In the case of the regularized model (3.1), (4.15), expressions for the corrections $r$ and $s$ were given by their first-order approximation (4.3).

\section{Appendix B. Three-dimensional regularized model}

$$
\begin{aligned}
\partial_{t} h & =-\partial_{x} q_{x}-\partial_{z} q_{z} \\
\delta \partial_{t} q_{x} & =\delta\left[\frac{9}{7} \frac{q_{x}^{2}}{h^{2}} \partial_{x} h-\frac{17}{7} \frac{q_{x}}{h} \partial_{x} q_{x}\right]+\left\{\frac{5}{6} h-\frac{5}{2} \frac{q_{x}}{h^{2}}+\delta\left[-\frac{8}{7} \frac{q_{x} \partial_{z} q_{z}}{h}-\frac{9}{7} \frac{q_{z} \partial_{z} q_{x}}{h}\right.\right.
\end{aligned}
$$




$$
\begin{aligned}
& \left.+\frac{9}{7} \frac{q_{x} q_{z} \partial_{z} h}{h^{2}}\right]+\eta\left[4 \frac{q_{x}\left(\partial_{x} h\right)^{2}}{h^{2}}-\frac{9}{2} \frac{\partial_{x} q_{x} \partial_{x} h}{h}-6 \frac{q_{x} \partial_{x x} h}{h}+\frac{9}{2} \partial_{x x} q_{x}+\frac{13}{4} \frac{q_{z} \partial_{x} h \partial_{z} h}{h^{2}}\right. \\
& -\frac{\partial_{z} q_{x} \partial_{z} h}{h}-\frac{43}{16} \frac{\partial_{x} q_{z} \partial_{z} h}{h}-\frac{13}{16} \frac{\partial_{z} q_{z} \partial_{x} h}{h}+\frac{3}{4} \frac{q_{x}\left(\partial_{z} h\right)^{2}}{h^{2}}-\frac{23}{16} \frac{q_{x} \partial_{z z} h}{h}-\frac{73}{16} \frac{q_{z} \partial_{x z} h}{h} \\
& \left.+\partial_{z z} q_{x}+\frac{7}{2} \partial_{x z} q_{z}\right]-\frac{5}{6} \zeta h \partial_{x} h+\frac{5}{6} h\left(\partial_{x x x}+\partial_{x z z} h\right\}\left(1-\frac{\delta}{70} q_{x} \partial_{x} h\right)^{-1}, \quad(\mathrm{~B} 1 b) \\
\delta \partial_{t} q_{z}= & \delta\left[\frac{9}{7} \frac{q_{z}^{2}}{h^{2}} \partial_{z} h-\frac{17}{7} \frac{q_{z}}{h} \partial_{z} q_{z}\right]-\frac{5}{2} \frac{q_{z}}{h^{2}}+\delta\left[-\frac{8}{7} \frac{q_{z} \partial_{x} q_{x}}{h}-\frac{9}{7} \frac{q_{x} \partial_{x} q_{z}}{h}+\frac{9}{7} \frac{q_{x} q_{z} \partial_{x} h}{h^{2}}\right] \\
& +\eta\left[4 \frac{q_{z}\left(\partial_{z} h\right)^{2}}{h^{2}}-\frac{9}{2} \frac{\partial_{z} q_{z} \partial_{z} h}{h}-6 \frac{q_{z} \partial_{z z} h}{h}+\frac{9}{2} \partial_{z z} q_{z}+\frac{13}{4} \frac{q_{x} \partial_{x} h \partial_{z} h}{h^{2}}-\frac{\partial_{x} q_{z} \partial_{x} h}{h}\right. \\
& -\frac{43}{16} \frac{\partial_{z} q_{x} \partial_{x} h}{h}-\frac{13}{16} \frac{\partial_{x} q_{x} \partial_{z} h}{h}+\frac{3}{4} \frac{q_{z}\left(\partial_{x} h\right)^{2}}{h^{2}}-\frac{23}{16} \frac{q_{z} \partial_{x x} h}{h}-\frac{73}{16} \frac{q_{x} \partial_{x z} h}{h}+\partial_{x x} q_{z} \\
& \left.+\frac{7}{2} \partial_{x z} q_{x}\right]-\frac{5}{6} \zeta h \partial_{z} h+\frac{5}{6} h\left(\partial_{x x z}+\partial_{z z z}\right) h .
\end{aligned}
$$

\section{Appendix C. Complete second-order model}

Writing $\varepsilon_{x}=1$ and $\varepsilon_{z}=0$, the complete second-order model consists of the evolution equations for $q_{x}, r_{x}$ and $s_{x}$ :

$$
\begin{aligned}
\delta \partial_{t} q_{x}= & \varepsilon_{x} \frac{27}{28} h-\frac{81}{28} \frac{q_{x}}{h^{2}}-33 \frac{r_{x}}{h^{2}}-\frac{3069}{28} \frac{s_{x}}{h^{2}}+\delta\left[-\frac{12}{5} \frac{q_{x} r_{x} \partial_{x} h}{h^{2}}-\frac{126}{65} \frac{q_{x} s_{x} \partial_{x} h}{h^{2}}+\frac{12}{5} \frac{r_{x} \partial_{x} q_{x}}{h}\right. \\
& +\frac{171}{65} \frac{s_{x} \partial_{x} q_{x}}{h}+\frac{12}{5} \frac{q_{x} \partial_{x} r_{x}}{h}+\frac{1017}{455} \frac{q_{x} \partial_{x} s_{x}}{h}+\frac{6}{5} \frac{q_{x}^{2} \partial_{x} h}{h^{2}}-\frac{12}{5} \frac{q_{x} \partial_{x} q_{x}}{h}-\frac{6}{5} \frac{q_{x} \partial_{z} q_{z}}{h} \\
& -\frac{6}{5} \frac{q_{z} \partial_{z} q_{x}}{h}+\frac{6}{5} \frac{q_{x} q_{z} \partial_{z} h}{h^{2}}-\frac{6}{5} \frac{q_{x} r_{z} \partial_{z} h}{h^{2}}-\frac{63}{65} \frac{q_{x} s_{z} \partial_{z} h}{h^{2}}-\frac{6}{5} \frac{q_{z} r_{x} \partial_{z} h}{h^{2}}-\frac{63}{65} \frac{q_{z} s_{x} \partial_{z} h}{h^{2}} \\
& +\frac{6}{5} \frac{r_{x} \partial_{z} q_{z}}{h}+\frac{108}{65} \frac{s_{x} \partial_{z} q_{z}}{h}+\frac{6}{5} \frac{r_{z} \partial_{z} q_{x}}{h}+\frac{63}{65} \frac{s_{z} \partial_{z} q_{x}}{h}+\frac{6}{5} \frac{q_{x} \partial_{z} r_{z}}{h}+\frac{576}{455} \frac{q_{x} \partial_{z} s_{z}}{h} \\
& \left.+\frac{6}{5} \frac{q_{z} \partial_{z} r_{x}}{h}+\frac{63}{65} \frac{q_{z} \partial_{z} s_{x}}{h}\right]+\eta\left[\frac{5025}{896} \frac{q_{x}\left(\partial_{x} h\right)^{2}}{h^{2}}-\frac{5055}{896} \frac{\partial_{x} q_{x} \partial_{x} h}{h}-\frac{10851}{1792} \frac{q_{x} \partial_{x x} h}{h}\right. \\
& +\frac{2027}{448} \partial_{x x} q_{x}+\partial_{z z} q_{x}-\frac{2463}{1792} \frac{\partial_{z} q_{x} \partial_{z} h}{h}+\frac{2433}{1792} \frac{q_{x}\left(\partial_{z} h\right)^{2}}{h^{2}}-\frac{5361}{3584} \frac{q_{x} \partial_{z z} h}{h} \\
& +\frac{7617}{1792} \frac{q_{z} \partial_{x} h \partial_{z} h}{h^{2}}-\frac{4749}{3584} \frac{\partial_{z} q_{z} \partial_{x} h}{h}-\frac{10545}{3584} \frac{\partial_{x} q_{z} \partial_{z} h}{h}-\frac{16341}{3584} \frac{q_{z} \partial_{x z} h}{h} \\
& \left.+\frac{1579}{448} \partial_{x z} q_{z}\right]-\frac{27}{28} \zeta h \partial_{x} h+\frac{27}{28} h\left(\partial_{x x x}+\partial_{x z z}\right) h,
\end{aligned}
$$

$$
\begin{aligned}
\delta \partial_{t} r_{x}= & \varepsilon_{x} \frac{1}{10} h-\frac{3}{10} \frac{q_{x}}{h^{2}}-\frac{126}{5} \frac{r_{x}}{h^{2}}-\frac{126}{5} \frac{s_{x}}{h^{2}}+\delta\left[\frac{1}{35} \frac{q_{x} \partial_{x} q_{x}}{h}-\frac{3}{35} \frac{q_{x}^{2} \partial_{x} h}{h^{2}}+\frac{108}{55} \frac{q_{x} r_{x} \partial_{x} h}{h^{2}}\right. \\
& -\frac{5022}{5005} \frac{q_{x} s_{x} \partial_{x} h}{h^{2}}-\frac{103}{55} \frac{r_{x} \partial_{x} q_{x}}{h}+\frac{9657}{5005} \frac{s_{x} \partial_{x} q_{x}}{h}-\frac{39}{55} \frac{q_{x} \partial_{x} r_{x}}{h}+\frac{10557}{10010} \frac{q_{x} \partial_{x} s_{x}}{h} \\
& -\frac{2}{35} \frac{q_{x} \partial_{z} q_{z}}{h}+\frac{3}{35} \frac{q_{z} \partial_{z} q_{x}}{h}-\frac{3}{35} \frac{q_{x} q_{z} \partial_{z} h}{h^{2}}+\frac{54}{55} \frac{q_{x} r_{z} \partial_{z} h}{h^{2}}+\frac{54}{55} \frac{q_{z} r_{x} \partial_{z} h}{h^{2}}-\frac{54}{55} \frac{r_{z} \partial_{z} q_{x}}{h} \\
& -\frac{54}{55} \frac{q_{z} \partial_{z} r_{x}}{h}-\frac{2511}{5005} \frac{q_{z} s_{x} \partial_{z} h}{h^{2}}-\frac{2511}{5005} \frac{q_{x} s_{z} \partial_{z} h}{h^{2}}+\frac{2511}{5005} \frac{s_{z} \partial_{z} q_{x}}{h}+\frac{2511}{5005} \frac{q_{z} \partial_{z} s_{x}}{h}
\end{aligned}
$$




$$
\begin{aligned}
& \left.-\frac{49}{55} \frac{r_{x} \partial_{z} q_{z}}{h}+\frac{7146}{5005} \frac{s_{x} \partial_{z} q_{z}}{h}+\frac{3}{11} \frac{q_{x} \partial_{z} r_{z}}{h}+\frac{1107}{2002} \frac{q_{x} \partial_{z} s_{z}}{h}\right]+\eta\left[\frac{93}{40} \frac{q_{x}\left(\partial_{x} h\right)^{2}}{h^{2}}\right. \\
& -\frac{69}{40} \frac{\partial_{x} h \partial_{x} q_{x}}{h}+\frac{21}{80} \frac{q_{x} \partial_{x x} h}{h}-\frac{9}{40} \partial_{x x} q_{x}-\frac{57}{80} \frac{\partial_{z} q_{x} \partial_{z} h}{h}+\frac{81}{80} \frac{q_{x}\left(\partial_{z} h\right)^{2}}{h^{2}}-\frac{3}{40} \frac{q_{x} \partial_{z z} h}{h} \\
& \left.+\frac{27}{80} \frac{q_{z} \partial_{x z} h}{h}+\frac{21}{16} \frac{q_{z} \partial_{x} h \partial_{z} h}{h^{2}}-\frac{63}{80} \frac{\partial_{z} q_{z} \partial_{x} h}{h}-\frac{9}{40} \frac{\partial_{z} h \partial_{x} q_{z}}{h}-\frac{9}{40} \partial_{x z} q_{z}\right] \\
& -\frac{1}{10} \zeta h \partial_{x} h+\frac{1}{10} h\left(\partial_{x x x}+\partial_{x z z} h,\right. \\
\delta \partial_{t} s_{x}= & \varepsilon_{x} \frac{13}{420} h-\frac{13}{140} \frac{q_{x}}{h^{2}}-\frac{39}{5} \frac{r_{x}}{h^{2}}-\frac{11817}{140} \frac{s_{x}}{h^{2}}+\delta\left[-\frac{4}{11} \frac{q_{x} r_{x} \partial_{x} h}{h^{2}}+\frac{18}{11} \frac{q_{x} s_{x} \partial_{x} h}{h^{2}}\right. \\
& -\frac{2}{33} \frac{r_{x} \partial_{x} q_{x}}{h}-\frac{19}{11} \frac{s_{x} \partial_{x} q_{x}}{h}+\frac{6}{55} \frac{q_{x} \partial_{x} r_{x}}{h}-\frac{288}{385} \frac{q_{x} \partial_{x} s_{x}}{h}-\frac{2}{11} \frac{q_{x} r_{z} \partial_{z} h}{h^{2}}-\frac{2}{11} \frac{q_{z} r_{x} \partial_{z} h}{h^{2}} \\
& +\frac{2}{11} \frac{r_{z} \partial_{z} q_{x}}{h}+\frac{2}{11} \frac{q_{z} \partial_{z} r_{x}}{h}+\frac{9}{11} \frac{q_{x} s_{z} \partial_{z} h}{h^{2}}+\frac{9}{11} \frac{q_{z} s_{x} \partial_{z} h}{h^{2}}-\frac{9}{11} \frac{s_{z} \partial_{z} q_{x}}{h}-\frac{9}{11} \frac{q_{z} \partial_{z} s_{x}}{h} \\
& \left.-\frac{8}{33} \frac{r_{x} \partial_{z} q_{z}}{h}-\frac{10}{11} \frac{s_{x} \partial_{z} q_{z}}{h}-\frac{4}{55} \frac{q_{x} \partial_{z} r_{z}}{h}+\frac{27}{385} \frac{q_{x} \partial_{z} s_{z}}{h}\right] \\
& +\eta\left[-\frac{3211}{4480} \frac{q_{x}\left(\partial_{x} h\right)^{2}}{h^{2}}+\frac{2613}{4480} \frac{\partial_{x} h \partial_{x} q_{x}}{h}-\frac{2847}{8960} \frac{q_{x} \partial_{x x} h}{h}+\frac{559}{2240} \partial_{x x} q_{x}+\frac{3029}{8960} \frac{\partial_{z} q_{x} \partial_{z} h}{h}\right. \\
& -\frac{3627}{8960} \frac{q_{x}\left(\partial_{z} h\right)^{2}}{h^{2}}+\frac{299}{17920} \frac{q_{x} \partial_{z z} h}{h}-\frac{559}{1792} \frac{q_{z} \partial_{x} h \partial_{z} h}{h^{2}}+\frac{4927}{17920} \frac{\partial_{z} q_{z} \partial_{x} h}{h} \\
& \left.-\frac{533}{17920} \frac{\partial_{x} q_{z} \partial_{z} h}{h}-\frac{5993}{17920} \frac{q_{z} \partial_{x z} h}{h}+\frac{559}{2240} \partial_{x z} q_{z}\right] \\
& -\frac{13}{420} \zeta h \partial_{x} h+\frac{13}{420} h\left(\partial_{x x x}+\partial_{x z z}\right) h, \\
& (\mathrm{C} 1 c)
\end{aligned}
$$

along with a symmetrical set of equations for $q_{z}, r_{z}$ and $s_{z}$, obtained from equations (C1) through the exchanges $\{x \leftrightarrow z\}$. The set of equations is then completed by the mass conservation $\partial_{t} h=-\partial_{x} q_{x}-\partial_{z} q_{z}$. The complete two-dimensional model is obtained by setting $\partial_{z} \equiv 0$ and $q_{z}=r_{z}=s_{z} \equiv 0$ in these equations.

\section{REFERENCES}

Alekseenko, S. V., Antipin, V. A., Guzanov, V. V., Kharlamov, S. M. \& Markovich, D. M. 2005 Three-dimensional solitary waves on falling liquid film at low Reynolds numbers. Phys. Fluids 17, 121704.

Alekseenko, S. V., Nakoryakov, V. Y. \& Pokusaev, B. G. 1994 Wave Flow of Liquid Films. Begell House.

Argyriadi, K., Serifi, K. \& Bontozoglou, V. 2004 Nonlinear dynamics of inclined films under low-frequency forcing. Phys. Fluids 16, 2457-2468.

Atherton, R. W. \& Homsy, G. M. 1976 On the derivation of evolution equations for interfacial waves. Chem. Engng Commun. 2, 57-77.

Balakotaiah, V. \& Mudunuri, R. R. 2004 Reply to comment on "Low-dimensional models for vertically falling viscous films". Phys. Rev. Lett. 93, 199402.

Balmforth, N. J. \& LiU, J. J. 2004 Roll waves in mud. J. Fluid Mech. 519, 33-54.

Benney, D. J. 1966 Long waves on liquid film. J. Math Phys. 45, 150-155.

Chang, H.-C. 1986 Traveling waves on fluid interfaces: Normal form analysis of the KuramotoSivashinsky equation. Phys. Fluids 29, 3142-3147.

Chang, H.-C. 1994 Wave evolution on a falling film. Annu. Rev. Fluid Mech. 26, 103-136. 
Chang, H.-C., Cheng, M., Demekhin, E. A. \& Kopelevitch, D. I. 1994 Secondary and tertiary excitation of three-dimensional patterns on a falling film. J. Fluid Mech. 270, 251-275.

Chang, H.-C., Demekhin, E. \& Kalaidin, E. 1996 Simulation of noise-driven wave dynamics on a falling film. AIChE J. 42, 1553-1568.

Chang, H.-C., Demekhin, E. A. \& Kopelevitch, D. I. 1993 Nonlinear evolution of waves on a vertically falling film. J. Fluid Mech. 250, 433-480.

Doedel, E. J., Champneys, A. R., Fairgrieve, T. F., Kuznetsov, Y. A., Sandstede, B. \& Wang, X.-J. 1997 Auto97: Continuation and bifurcation software for ordinary differential equations. Tech. Rep. Department of Computer Science, Concordia University, Montreal, Canada (available by FTP from ftp.cs. concordia.ca in directory pub/doedel/auto).

Floryan, J., Davis, S. \& Kelly, R. 1987 Instabilities of a liquid film flowing down a slightly inclined plane. Phys. Fluids 30, 983-989.

GJEviK, B. 1970 Occurence of finite-amplitude surface waves on falling liquid films. Phys. Fluids 13, 1918-1925.

GJEviK, B. 1971 Spatially varying finite-amplitude wave trains on falling liquid films. Acta Polytech. Scand. Me. 61, 1-16.

GLENDinning, P. \& SPARrow, C. 1984 Local and global behaviour near homoclinic orbits. J. Statist. Phys. 35, 645-696.

Herbert, T. 1988 Secondary instability of boundary layers. Annu. Rev. Fluid Mech. 20, 487.

Joo, S. \& Davis, S. 1992 Instabilities of three-dimensional theory viscous falling films. J. Fluid Mech. 242, 529 .

Kapitza, P. L. 1948 Wave flow of thin layers of a viscous fluid. In Collected Papers of P.L. Kapitza (ed. D. T. Haar), pp. 662-689. Pergamon, original paper: Zh. Ekper. Teor. Fiz. 18, 3-28 (in Russian).

Kapitza, P. L. \& Kapitza, S. P. 1949 Wave flow of thin layers of a viscous fluid. In Collected Papers of P.L. Kapitza (ed. D. T. Haar), pp. 690-709. Pergamon, original paper: Zh. Ekper. Teor. Fiz. 19, 105-120 (in Russian).

Lin, S. P. 1974 Finite amplitude side-band stability of a viscous fluid. J. Fluid Mech. 63, 417-429.

LiU, J. \& Gollub, J. P. 1993 Onset of spatially chaotic waves on flowing films. Phys. Rev. Lett. 70, $2289-2292$.

LiU, J. \& Gollub, J. P. 1994 Solitary wave dynamics of film flows. Phys. Fluids 6, 1702-1712.

LiU, J., Paul, J. D. \& Gollub, J. P. 1993 Measurements of the primary instabilities of film flows. J. Fluid Mech. 250, 69-101.

Liu, J., Schneider, J. B. \& Gollub, J. P. 1995 Three-dimensional instabilites of film flows. Phys. Fluids 7, 55-67.

Malamataris, N. A., Vlachogiannis, M. \& Bontozoglou, V. 2002 Solitary waves on inclined films: Flow structure and binary interactions. Phys. Fluids 14, 1082-1094.

NAKaYA, C. 1975 Long waves on a thin fluid layer flowing down an inclined plane. Phys. Fluids 18, $1407-1412$.

Nosoko, T. \& Miyara, A. 2004 The evolution and subsequent dynamics of waves on a vertically falling liquid film. Phys. Fluids 16, 1118-1126.

Nosoko, T., Yoshimura, P. N., Nagata, T. \& OKawa, K. 1996 Characteristics of two-dimensional waves on a falling liquid film. Chem. Engng Sci. 51, 725-732.

Ooshida, T. 1999 Surface equation of falling film flows with moderate Reynolds number and large but finite Weber number. Phys. Fluids 11, 3247-3269.

Oron, A. \& Gottlieb, O. 2004 Subcritical and supercritical bifurcations of the first- and second order Benney equations. J. Engng Maths 50, 121-140.

Panga, M. K. R. \& Balakotaiah, V. 2003 Low-dimensional models for vertically falling viscous films. Phys. Rev. Lett. 90, 154501.

Panga, M. K. R., Mudunuri, R. R. \& Balakotaiah, V. 2005 Long-wavelength equation for vertically falling films. Phys. Rev. E 71, 036310.

PARK, C. D. \& Nosoko, T. 2003 Three-dimensional wave dynamics on a falling film and associated mass transfer. AIChE J. 49, 2715-2727.

Press, W., Teukolsky, S., Vetterling, W. \& Flannery, B. 1992 Numerical Recipes in C-The Art of Scientific Computing, 2nd edn. Cambridge University Press.

Pumir, A., Manneville, P. \& Pomeau, Y. 1983 On solitary waves running down an inclined plane. J. Fluid Mech. 135, 27-50. 
Ramaswamy, B., Chippada, S. \& Joo, S. W. 1996 A full-scale numerical study of interfacial instabilities in thin-film flows. J. Fluid Mech. 325, 163-194.

Roberts, A. 1996 Low-dimensional models of thin film fluid dynamics. Phys. Lett. A 212, 63-71.

Roskes, G. 1969 Three-dimensional long waves on a liquid film. Phys. Fluids 13, 1440-1445.

Roy, R., Roberts, A. \& Simpson, M. 2002 A lubrication model of coating flows over a curved substrate in space. J. Fluid Mech. 454, 235-261.

RuYer-Quil, C. 1999 Dynamique d'un film mince s'écoulant le long d'un plan incliné. PhD dissertation, École polytechnique.

Ruyer-Quil, C. \& Manneville, P. 1998 Modeling film flows down inclined planes. Eur. Phys. J. B 6, 277-292.

Ruyer-Quil, C. \& Manneville, P. 2000 Improved modeling of flows down inclined planes. Eur. Phys. J. B 15, 357-369.

Ruyer-Quil, C. \& Manneville, P. 2004 Comment on "Low-dimensional models for vertically falling viscous films". Phys. Rev. Lett 93, 199401.

Ruyer-Quil, C., Scheid, B., Kalliadasis, S., Velarde, M. G. \& R. Kh. Zeytounian 2005 Thermocapillary long waves in a liquid film flow. Part 1. Low-dimensional formulation. J. Fluid Mech. 538, 199-222.

Salamon, T., Armstrong, R. \& Brown, R. 1994 Traveling waves on vertical films: Numerical analysis using the finite element method. Phys. Fluids 6, 2202-2220.

Saprykin, S., Demekhin, E. A. \& Kalliadasis, S. 2005 Self-organization of two-dimensional waves in an active-dispersive-dissipative nonlinear medium. Phys. Rev. Lett. 94, 224101.

Scheid, B., Ruyer-Quil, C., Kalliadasis, S., Velarde, M. G. \& R. Kh. Zeytounian $2005 a$ Thermocapillary long waves in a liquid film flow. Part 2. Linear stability and nonlinear waves. J. Fluid Mech. 538, 223-244.

Scheid, B., Ruyer-Quil, C., Thiele, U., Kabov, O., Legros, J. \& Colinet, P. $2005 b$ Validity domain of the Benney equation including Marangoni effect for closed and open flows. J. Fluid Mech. 527, 303-335.

Schlichting, H. 1955 Boundary-Layer Theory. McGraw-Hill.

Schmid, P. J. \& Henningson, D. S. 2001 Stability and Transition in Shear Flows. Springer.

SHKadov, V. 1967 Wave flow regimes of a thin layer of viscous fluid subject to gravity. Izv. Ak. Nauk SSSR, Mekh. Zhi. Gaz 2, 43-51 (English transl. Fluid Dynamics 2, Faraday Press, NY, 1970, pp. 29-34).

ShKadov, V. 1977 Solitary waves in a layer of viscous liquid. Izv. Ak. Nauk SSSR, Mekh. Zhi. Gaz $1,63-66$.

Tihon, J., Tovchigrechko, V., Sobol'ik, V. \& Wein, O. 2003 Electrodiffusion detection of the near-wall flow reversal in liquid films at the regime of solitary waves. J. Appl. Electrochem. 33 (7), 577-587.

TRIfonov, Y. Y. 1989 Bifurcations of two-dimensional into three-dimensional wave regimes for a vertically flowing liquid film. Izv. Ak. Nauk SSR, Mekh. Zh. Gaza 5, 109-114.

Vlachogiannis, M. \& Bontozoglou, V. 2001 Observations of solitary wave dynamics of film flows. J. Fluid Mech. 435, 191.

Whitham, G. B. 1974 Linear and Nonlinear Waves. Wiley-Interscience. 